\title{
The Geometry of the Hyperbolic System for an Anisotropic Perfectly Elastic Medium
}

\author{
Dirk-J. Smit' ${ }^{1}$, Maarten V. de Hoop ${ }^{2}$ \\ ${ }^{1}$ Koninklijke/Shell Exploratie en Produktie Laboratorium, Volmerlaan 6, 2288 GD Rijswijk ZH, \\ The Netherlands \\ ${ }^{2}$ Schlumberger Cambridge Research, High Cross, Madingley Road, Cambridge CB3 0EL, England
}

Received: 17 August 1993/In revised form: 19 January 1994

\begin{abstract}
We evaluate the fundamental solution of the hyperbolic system describing the generation and propagation of elastic waves in an anisotropic solid by studying the homology of the algebraic hypersurface defined by the characteristic equation, also known as the "slowness" surface. Our starting point is the Herglotz-PetrovskyLeray integral representation of the fundamental solution. We find an explicit decomposition of the latter solution into integrals over vanishing cycles associated with the isolated singularities on the slowness surface. As is well known in the theory of isolated singularities, integrals over vanishing cycles satisfy a system of differential equations known as Picard-Fuchs equations. Such equations are linear and can have at most regular singular points. We discuss a method to obtain these equations explicitly. Subsequently, we use the monodromy properties around the regular singular points to find the asymptotic behavior according to the different types of singularities that may appear on a wave front in three dimensions. This is a method alternative to the one that arises in the Maslov theory of oscillating integrals. Our work sheds new light on how to compute and classify the CagniardDe Hoop contour in the complex radial horizontal slowness plane; this contour is used in numerical integration schemes to obtain the full time behaviour of the fundamental solution for a given direction of propagation.
\end{abstract}

\section{Introduction}

Over the last few years, much attention has been paid to the evaluation of the fundamental solution (Green's tensor) of the hyperbolic system describing the generation and propagation of waves in anisotropic solids in $n=3$-dimensional space. One reason for this comes from the field of exploration geophysics; recently developed techniques in seismic acquisition and processing are powerful enough to reveal, in principle, anisotropic properties of rock in layers at great depth. Knowledge of this anisotropy is important to the oil and gas industries as, on the one hand some of the anisotropy in layered hydrocarbon reservoirs is due to large joint systems (faults and fractures), which will affect the fluid and gas flows, while on the other hand 
the caprock of the reservoir is often a shale, the degree of anisotropy of which may be related to its sealing potential.

Wave propagation in anisotropic media is very different from propagation in isotropic media. Generic for anisotropic media are the phenomena of shear-wave splitting and conical refraction. In addition, several types of singularities appear, like the self intersections, cusps and swallowtails that a shear-wave front develops. The occurrence of a singularity affects the solution significantly; near a singularity the leading order time behavior cannot be described by standard so-called asymptotic ray theory. The latter approximation diverges in the vicinity of a singularity. As the physical solution at a wave front singularity is still regular, more powerful theories have been developed to find this solution. (The understanding of the time behavior near a singularity is crucial to interpret it properly on measured seismograms.) A geometrical description of waves leads to a generally valid solution of the hyperbolic system, which is the approach we will follow in this paper.

Different approaches to solve a 3-dimensional anisotropic hyperbolic system, all of them based on plane-wave expansions of the particle velocity or the Lamé potentials, have been explored to find closed-form integral representations of the solution. We mention the Sommerfeld-Weyl representation [42], Riesz's method [39], and the Herglotz-Petrovsky-Leray (HPL) representation [11, 38]. The reduction of the number of integrals in the latter representation was independently achieved, employing the time-Laplace transform domain, in the Cagniard-De Hoop method [13, 20, 24]. For particular symmetries the fundamental solution could be found explicitly (e.g., the work of Payton [36] and of Burridge, Chadwick and Norris [12]). In crystal acoustics (e.g., Musgrave [32]) and phonon focusing (see Every [17]) similar developments took place. However, most of these techniques lack a precise geometrical understanding of the features typical for the wave solution in anisotropic media.

The HPL formula gives the fundamental solution in the form of an Abelian integral of a rational closed $(n-1)$-form integrated over a complex $(n-1)$-dimensional algebraic hypersurface [8]. From the latter integral the full Green's tensor of the problem can be constructed. The algebraic hypersurface, known as the slowness surface, is defined by the equation $H(\xi)=0$ of degree $D=2 n$, the polynomial $H$ being the complexified determinant of the symbol matrix of the hyperbolic operator. The integral is defined over a tube $\gamma$ of properly oriented cycles $\partial \gamma$ on the slowness surface. The general form of the integral is

$$
E(x, t)=\int_{\gamma(x, t)} \frac{d \xi_{1} \ldots d \xi_{n-1}}{\partial_{\xi_{n}} H},
$$

where $\xi_{n}$ is the coordinate in slowness space chosen along the direction of propagation of interest. The boundary of $\gamma$, denoted by $\partial \gamma$, depends on a parameter $t$ which can be identified with time. The $(n-2)$-cycle $\partial \gamma$ is shown to be related to the so-called Cagniard-De Hoop contour.

The theory of integrals like (1.1) is extensive and well established in the mathematical literature. In particular, its frequency-domain counterpart is properly treated by the Maslov theory of rapidly oscillating integrals [16], which is also valid near the wave front singularities [8]. In this paper, we employ algebraic-geometrical techniques, known in complex singularity theory, to analyse integrals like (1.1) with the application to 3-dimensional wave propagation in an anisotropic, perfectly elastic medium in mind. In particular, we analyse the asymptotic behavior of the solution 
near those parts of the wave fronts corresponding to degenerate critical points on the slowness surface. Such points are referred to as singularities. The propagation of wave fronts in the vicinity of critical points at which conical refraction occurs, has been addressed recently in [10] where also an application to generic elastic media is mentioned.

Our line of thought is different from the usual Maslov theory approach, in that we will write the solution of the hyperbolic problem as a linear combination of certain contour integrals uniquely associated with the polynomial functions $f$, say, parametrizing the slowness surface around its critical points. The contours are in fact not defined on the slowness surface itself, but on a non-singular level surface of the above mentioned function $f$. These non-singular level surfaces follow from the zero locus of a function $F(\xi, x)$ defined on the phase space (with coordinates $(\xi, x))$ with the property that $F(\xi, 0)=f(\xi)$. The contours in the phase space are also called the vanishing cycles; they are associated with the singularity on the slowness surface. Their name originates from the (defining) property that these cycles skrink to a point upon projection to the slowness surface (taking all relevant physical space coordinates zero, i.e., in the limit $F \rightarrow f$ ). Mathematically, the space coordinates $x$ are the deformation parameters of the singularity defined by $f$. Near a singularity the deformed slowness surface can be written as the zero locus of a quasihomogeneous polynomial function $(F)$ in suitable, so-called normal coordinates. For each different type of critical point there is a different polynomial function. The parameters $x$ lift the degeneracy of the critical point $f$ into several non-degenerate critical points. It often turns out that it is not strictly necessary to use all the $x$ coordinates to lift this degeneracy: a subset already does the job. This subset of parameters can be shown to correspond to the so-called versal parameters denoted by $\lambda$ playing a central rôle in singularity theory. We will show explicitly how the versal parameters $\lambda$ may be introduced to replace the parameters $x$. In summary, we shall rewrite the fundamental solution near a singularity on the slowness surface as a linear combination of integrals over vanishing cycles associated with the versal deformations of singularity. This is a somewhat unconventional approach in the analysis hyperbolic differential equations.

It is well known in singularity theory that the vanishing cycles are not uniquely defined by the type of the singularity. Combined with the fact that the integrand is a closed rational form of maximum degree (it is a volume form) this leads to the observation that the integral over a vanishing cycle satisfies a system of linear differential equations in the versal parameters of the singularity. These linear differential equations are known as the Picard-Fuchs equations. They can have at most regular-singular points, hence an asymptotic analysis close to the points on the wave front corresponding to the critical points on the slowness surface is in principle straightforward. It is one of the objectives of this paper to produce an elementary algorithm to derive the Picard-Fuchs system from the local polynomial function $F(\xi, 0)$ parametrizing the slowness surface in a neighborhood of any singularity.

However, if we had chosen a different basis in the space of vanishing cycles, the coefficients in the linear decomposition into integrals over vanishing cycles would have been different. Furthermore, the Picard-Fuchs system would have changed. However, the solution at the wave front is invariant under a reparameterization of the vanishing cycles. Mathematically, this means that the integral should be expanded in an integral homology basis. It has only recently been understood, though in a different context [28], that such invariance can be reflected in the basis 
of cycles by studying the invariants of the Picard-Fuchs equations. These invariants are built out of particular quotients of the solutions of the Picard-Fuchs equations, see the references in [28]. (A solution of a linear differential equation that does not change under a monodromy transformation around its regular singular points is called an invariant.) In other words, a specific choice of basis cycles implies a specific parametrization of the level surfaces. The transformation of the initially arbitrarily chosen versal parameters to the specific ones follow from the invariants of the Picard-Fuchs system.

In this paper we show how the freedom in the parametrization of the vanishing cycles is of use in the study of the hyperbolic equation governing waves in elastic media. It follows that one can compute the coefficients in the linear expansion into vanishing cycles when they are invariantly parametrized. The formula for these coefficients has been derived before by V.A. Vasiliev. However, the connection with the invariants of the Picard-Fuchs system is new. The advantage of the understanding of this connection is that it shows explicitly the geometrical content of the solution near the singular parts of the wave front (which coincides with the high-frequency limit). This part of the solution is "topological" in that it is fixed by the type of the singularity. Furthermore, the decomposition into vanishing cycles is also valid away from the singularity. This is interesting in itself, as it implies a canonical representation of the so-called Cagniard-De Hoop contours which may be relevant in (future) numerical schemes to evaluate $E$ close to the wave front. The full solution $E$ obtained after integration over the full slowness surface is completely fixed (up to analytical functions) by the solution of the Picard-Fuchs system for each critical point. Thus a classification of the types of possible critical points on a generic slowness surface leads to a classification of possible solutions $E$ near the associated wave fronts.

The rôle of isolated singularities for wave front geometries has been extensively studied in the mathematical literature, most notably by V.I. Arnold (see [7] for a recent overview). The relation between a cycle $\partial \gamma$ and certain objects in singularity theory has been explored before by Vasiliev [43] and in fact been foreseen by Petrovsky [38], where the cycle was described in terms of a certain cohomology class, at present called the Petrovsky class, in relation with the recognition of lacunae in the fundamental solution of a hyperbolic operator. We will reestablish this relation, using the approach followed by Atiyah, Bott and Gårding [8].

The paper is organised as follows. In Sect. 2 we pose the seismic Cauchy problem and recall some of its basic properties. In Sect. 3 we derive explicitly the HPL representation for a general elastodynamic hyperbolic system from Gelfand's plane-wave expansion $[18,25]$. Along the way we discuss the geometrical properties of the slowness surface relevant to the later analysis. In Sect. 3 we also show how the HPL formula reduces to an integral over a $(n-2)$-dimensional cycle on the slowness surface, which can be associated with the Cagniard-De Hoop contour. In Sect. 4 the latter integral is decomposed into integrals over vanishing cycles associated with the singularities. Introducing the appropriate parametrization, we compute the coefficients in the expansion of the original integral. In Sect. 5, finally, we derive a Picard-Fuchs differential equation for the integrals over vanishing cycles and show how the asymptotic behavior in the high-frequency limit follows from the monodromy properties around the regular singular points. 


\section{The Cauchy Problem}

The Tensorial Cauchy Problem. In this section we introduce the tensorial Cauchy problem arising in seismics. We define the physical quantities and transform the tensorial problem into an associated scalar Cauchy problem, from which the full Green's tensor can be constructed. The scalar problem is completely equivalent to the tensorial problem but is more suitable for our analysis. Since the procedure to arrive at this scalar Cauchy problem is not so well known, though standard in functional analysis, we will present the relevant steps explicitly.

It is assumed that linear elasticity theory is applicable. Then the particle velocity $v_{m}, m=1, \ldots, n$ satisfies the system of equations

$$
\left[\delta_{k m} \hat{o}_{t}^{2}-\rho^{-1} \hat{o}_{r} c_{k r m s} \hat{o}_{s}\right] v_{m}=\rho^{-1}\left[\hat{\partial}_{t} f_{k}+\hat{\partial}_{r}\left(c_{k r m s} h_{m s}\right)\right],
$$

where the summation convention applies and

$$
\begin{aligned}
\rho & =\text { volume density of mass, } \\
c_{k r m s} & =\text { stiffness } \\
f_{k} & =\text { volume source density of force, } \\
h_{m s}=h_{s m} & =\text { volume source density of deformation rate. }
\end{aligned}
$$

Note that $h_{m s}$ is related to the seismic moment density tensor, $m_{k r}$ say, according to

$$
\left(\hat{o}_{t} m_{k r}\right) \delta\left(n_{j} x_{j}\right)=c_{k r m s} h_{m s},
$$

where $n_{j}$ is the unit normal to the plane of dislocation. The $(n \times n)$ principal part of the tensorial wave operator on the left-hand side of (2.1) is given by

$$
\delta_{k m} \hat{o}_{t}^{2}-C_{k m}^{r s} \hat{\partial}_{r} \hat{\partial}_{s}
$$

with

$$
C_{k m}^{r s}=\rho^{-1} c_{k r m s} .
$$

From the positive definite property of the strain-energy function it follows that the system is hyperbolic, that is, the system admits propagating waves as solutions; in general, the system is not strictly hyperbolic, which means that different modes may propagate with the same speed in particular directions. The symmetries of the stiffness yield

$$
C_{k m}^{r s}=C_{k s}^{r m}=C_{r m}^{k s}=C_{m k}^{s r}=C_{r s}^{k m} .
$$

They follow from the condition that angular momentum is conserved and from the assumption that the processes of deformation are adiabatic. The macroscopic symmetry properties of the medium, collected in the "point" group of transformations $O$, say, simply reflect themselves in the relations $O_{k^{\prime} k} O_{m^{\prime} m} O_{r^{\prime} r} O_{s^{\prime} s} C_{m^{\prime} k^{\prime}}^{s^{\prime} r^{\prime}}=C_{k m}^{s r}$. How to obtain the tensor $C$ from microstructure of rocks can be found in several papers amongst which the ones by Hudson [23], Schoenberg and Muir [41] and Nichols, Muir and Schoenberg [34].

For seismic applications the Green's tensor is introduced as the particle velocity due to a point body force (e.g., a vibrator) with signature $H(t)$ (the Heaviside function; this yields a $\delta$ behavior in time on the right-hand side of (2.1)). For 
mathematical convenience a linear combination of the causal and the anti-causal Green's tensor is taken to constitute the fundamental solution; the latter reduces to the causal Green's tensor on the positive real time axis. Thus, using Duhamel's principle [25], the tensorial Cauchy problem to construct the fundamental solution is introduced: determine the solution $g_{m}$ of the homogeneous tensorial wave equation

$$
\left[\delta_{k m} \hat{o}_{t}^{2}-C_{k m}^{r s} \hat{\partial}_{r} \hat{o}_{s}\right] g_{m}=0 \text { for }\{x, t\} \in \mathbb{R}^{n+1},
$$

satisfying the initial conditions

$$
\left.g_{m}\right|_{t=0}=0,\left.\hat{o}_{t} g_{m}\right|_{t=0}=a_{m} \delta\left(x_{1}, \ldots, x_{n}\right) .
$$

By taking for $a_{m}$ the standard unit vectors, the columns of the fundamental solution are found. Later on, we will ignore the separation between the space and time coordinates and set $\{x, t\} \rightarrow\left\{x_{1}, \ldots, x_{n+1}\right\} \in \hat{Z}^{\prime}$.

Through mutual elimination of the components of the system (2.6), it follows that any component $g_{m}$ satisfies the $D=2 n$-degree equation in $n$ dimensions

$$
a\left(\partial_{i}, \partial_{t}\right) g_{m}=0 \text {, }
$$

in which

$$
a\left(\hat{o}_{i}, \hat{\partial}_{t}\right)=\operatorname{det}\left[\delta_{k m} \hat{\partial}_{t}^{2}-C_{k m}^{r s} \partial_{r} \partial_{s}\right]
$$

with initial conditions

$$
\left.\partial_{t}^{\prime} g_{m}\right|_{t=0}=\phi_{m k}^{(\prime)} a_{k}, \ell=0, \ldots, 2 n-1,
$$

where, using our wave operator repeatedly, $\phi_{m k}^{(\text {even })}=0$ and

$$
\phi_{m k}^{\left(2 / 0^{-1}\right)}=\left(\left[C^{r s} \partial_{r} \partial_{s}\right]^{\prime 0}\right)_{m k} \delta\left(x_{1}, \ldots, x_{n}\right), \ell_{0}=1, \ldots, n
$$

for $m=1, \ldots, n$.

The Scalar Cauchy Problem. Now, consider the particular family of scalar Cauchy problems

$$
a\left(\partial_{i}, \hat{\partial}_{t}\right) E^{(/)}=0
$$

with

$$
\left.\partial_{t}^{\prime^{\prime}} E^{(\prime)}\right|_{t=0}=\delta_{\ell^{\prime},} U\left(x_{1}, \ldots, x_{n}\right), \ell^{\prime}=0, \ldots, 2 n-1
$$

for $\ell=0, \ldots, 2 n-1$. The solution is denoted by $E^{(/)}[U]$ to indicate the initial value function explicitly. Note that for $\ell=0, \ldots, 2 n-2$,

$$
E^{(\prime)}[U]=\partial_{t}^{2 n-1-(} E^{(2 n-1)}[U]-\sum_{\ell^{\prime}=\ell+1}^{2 n-1} E^{\left(\prime^{\prime}\right)}\left[\partial_{t}^{\ell^{\prime}-(} \partial_{t}^{2 n-1} E^{(2 n-1)}[U]\right] .
$$

This way, the $E^{(/)}, \ell=0, \ldots, 2 n-2$, follow from $E^{(2 n-1)}$ by recursion. The further analysis will be focussed on the latter function. Thus, set

$$
E=E^{(2 n-1)}[\delta]
$$

then

$$
E^{(2 n-1)}[U]=E * \mathbb{R}^{n} U
$$

The $*_{\mathbb{R}^{n}}$ denotes convolution with $U$ in $\mathbb{R}^{n}$. Note that the "physical" scalar Cauchy problem is associated with $E^{(1)}$. 
The function $g_{m}$ can now be constructed from the $E^{(/)}$using (2.10):

$$
g_{m}=a_{k}\left\{\sum_{/ 0=1}^{n} \Phi_{m k}^{\left(2 / 0^{-1)}\right.}\left(E^{\left(2 / 0^{-1}\right)}[\delta]\right)\right\}
$$

where

$$
\Phi_{m k}^{\left(2 / 0^{-1)}\right.}(.)=\phi_{m k}^{\left(2 / 0^{-1)}\right.} * \mathbb{R}^{n},, \ell_{0}=1, \ldots, n .
$$

In seismic applications the $n=3$-dimensional problem is considered, although the 2-dimensional problem arises as well namely from propagation in a plane of symmetry in three dimensions. In that case a line source rather than a point source is considered.

Gel'fand's Plane-Wave Expansion. We will now employ Gel'fand's plane-wave expansion (the inversion formula for the Radon transformation) of the $\delta$-source in the Cauchy problem to arrive at a plane-wave expansion of the fundamental solution. We will employ explicit distributions on $\mathbb{R}$ which are boundary values of functions analytic in the lower or upper complex half-plane. According to Gel'fand [18], we have

$$
\begin{aligned}
\delta\left(x_{1}, \ldots, x_{n}\right) & =-\frac{i^{n-1}}{2^{n} \pi^{n-1}} \int_{\Omega} \Delta^{(n-1)}\left(\hat{\xi}_{j} x_{j}-i 0\right) \mathrm{d} S(\hat{\xi}) \\
& =\frac{i^{n-1}}{2^{n} \pi^{n-1}} \int_{\Omega}^{\Delta^{(n-1)}\left(\hat{\xi}_{j} x_{j}+i 0\right)} \mathrm{d} S(\hat{\xi}),
\end{aligned}
$$

where

$$
\Delta(\tau)=\frac{i}{\pi \tau}
$$

is the analytic Dirac distribution (odd in $\tau$ ), $\Omega$ is the unit sphere and $\mathrm{d} S(\hat{\xi})$ is the volume form on this sphere. To employ this expansion in our initial value problem, we introduce the family of analytic functions

$$
\chi_{z}(\tau)=\Gamma(-z) \frac{\exp (-i \pi z)}{i \pi} \tau=
$$

for $z \in \mathbb{C} \backslash\{0,1,2, \ldots\}$. (Note that $\Gamma$ has simple poles at $0,-1,-2, \ldots$ ) This is a single-valued analytic function in the lower half-plane $(\operatorname{Im}\{\tau\}<0)$ but likewise it is a single-valued function in the upper half-plane $(\operatorname{Im}\{\tau\}>0)$. This family of functions satisfies the relation

$$
\frac{\mathrm{d} \chi z}{\mathrm{~d} \tau}=\chi_{z-1}(\tau)
$$

The analytic Dirac distribution is contained as a member in the family:

$$
\chi-1(\tau)=\Delta(\tau)
$$

Using the Laurent series of (2.21) in $z$ about the simple poles at $z=0,1,2, \ldots, \chi z$ can be defined for positive integer values for $z$ as follows. Let $m=0,1,2, \ldots$, then

$$
\left.\chi_{m}(\tau) \rightarrow \frac{\mathrm{d}}{\mathrm{d} \zeta} \zeta \chi_{m+\zeta}(\tau)\right|_{\zeta=0}=\frac{1}{m !}\left[1-\frac{i}{\pi}\left(\log \left(\tau^{-1}\right)+c_{m}\right)\right] \tau^{m}
$$


with

$$
c_{0}=\Gamma^{\prime}(1), c_{m}=c_{0}+\sum_{k=1}^{m} \frac{1}{k} \text { for } m=1,2, \ldots .
$$

The expression in (2.24) satisfies (2.22) with $z=m$. Further, it is found that

$$
\begin{aligned}
& \lim _{\operatorname{Im}\{\tau\} \downarrow 0} \chi_{m}(\tau)=\frac{1}{m !}\left[\frac{1}{2} \operatorname{sgn}(\tau)+\frac{1}{2}-\frac{i}{\pi}\left(\log \left(|\tau|^{-1}\right)+c_{m}\right)\right] \tau^{m}, \\
& \lim _{\operatorname{Im}\{\tau\} \uparrow 0} \chi_{m}(\tau)=\frac{1}{m !}\left[-\frac{1}{2} \operatorname{sgn}(\tau)+\frac{3}{2}-\frac{i}{\pi}\left(\log \left(|\tau|^{-1}\right)+c_{m}\right)\right] \tau^{m},
\end{aligned}
$$

for $m=0,1,2, \ldots$ Now, using (2.22) for $m=0$, we find

$$
\begin{aligned}
& \lim _{\operatorname{Im}\{\tau\} \downarrow 0} \Delta(\tau)=\lim _{\operatorname{Im}\{\tau\} \downarrow 0} \chi-1(\tau)=\delta(\tau)+i(\mathscr{H} \delta)(\tau), \\
& \lim _{\operatorname{Im}\{\tau\} \uparrow 0} \Delta(\tau)=\lim _{\operatorname{Im}\{\tau\} \uparrow 0} \chi-1(\tau)=-\delta(\tau)+i(\mathscr{H} \delta)(\tau),
\end{aligned}
$$

where $\mathscr{H}$ denotes the Hilbert transform.

Explicit evaluation reveals that

$$
\begin{aligned}
& \int_{\Omega} \operatorname{Re}\left\{\chi_{-n}\left(\hat{\xi}_{j} x_{j}-i 0\right)\right\} \mathrm{d} S(\hat{\xi})=0 \text { if } n \text { is even, } \\
& \int_{\Omega} \operatorname{Im}\left\{\chi_{-n}\left(\hat{\xi}_{j} x_{j}-i 0\right)\right\} \mathrm{d} S(\hat{\xi})=0 \text { if } n \text { is odd }
\end{aligned}
$$

Thus, in fact (2.19) can be written as

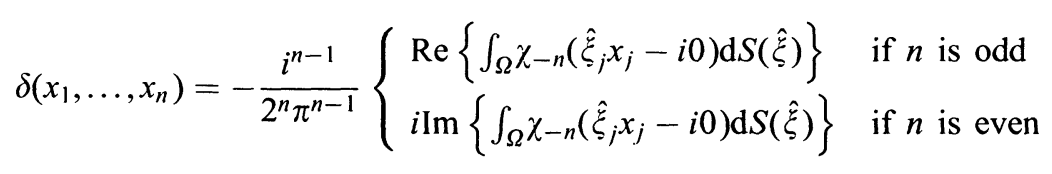

or

$$
\delta\left(x_{1}, \ldots, x_{n}\right)=-\frac{1}{2^{n} \pi^{n-1}} \operatorname{Re}\left\{\int_{\Omega} i^{n-1} \chi_{-n}\left(\hat{\xi}_{j} x_{j}-i 0\right) \mathrm{d} S(\hat{\xi})\right\},
$$

but everywhere $\chi_{-n}\left(\hat{\xi}_{j} x_{j}-i 0\right)$ can be replaced by

$$
(1 / 2)\left[\chi-n\left(\hat{\xi}_{j} x_{j}-i 0\right)-\overline{\chi-n\left(\hat{\xi}_{j} x_{j}+i 0\right)}\right] .
$$

Given Gel'fand's plane-wave expansion of the $\delta$-function, it is now straightforward to find the plane-wave expansion both of $g_{m}$ in case of the tensorial problem and of $E$ in case of the scalar problem. We will suppress the term $-i 0$ in all the arguments to simplify our notation.

The plane-wave expansion of $g_{m}$ yields:

$$
g_{m}=-\frac{i^{n-1}}{2^{n} \pi^{n-1}} \sum_{p<0, p>0} \int_{\Omega} \alpha^{(p)}(\hat{\xi}) e_{m}^{(p)}(\hat{\xi}) \chi_{-n+1}\left(\hat{\xi}_{j} x_{j}-\lambda_{p}(\hat{\xi}) t\right) \mathrm{d} S(\hat{\xi}) .
$$

Here, the $\lambda_{p}, p \in\{ \pm 1, \pm 2, \ldots, \pm n\}$ are the phase velocities that must satisfy the dispersion relation

$$
a\left(\hat{\xi}_{i},-\hat{\lambda}_{p}\right)=0,
$$


while the $e^{(p)}$ are the polarization vectors corresponding with the particle velocity; they satisfy the Christoffel equation

$$
\mathscr{K}_{k m} e_{m}^{(p)}=\lambda_{p}^{2} e_{k}^{(p)},
$$

where

$$
\mathscr{K}_{k m}=C_{k m}^{r s} \hat{\xi}_{r} \hat{\xi}_{s}
$$

is the Christoffel matrix. Since this matrix is symmetric, the basis $\left\{e^{(p)}\right\}_{p>0}$ can be chosen to be orthonormal at every $\hat{\xi}$. Later on, we will group together the spectral-domain coordinates: $\left\{\hat{\xi}_{1}, \ldots, \hat{\xi}_{n},-\hat{i}\right\} \rightarrow\left\{\hat{\xi}_{1}, \ldots, \hat{\xi}_{n+1}\right\} \in \hat{Z}$, say. In view of the symmetry under time reversal of the tensorial wave operator, we have

$$
\lambda_{-p}=-\lambda_{p}
$$

and we can choose

$$
e^{(-p)}=e^{(p)} .
$$

Hence, (2.35) must be polynomial in $\hat{i}_{p}^{2}$; if $n=3$ the equation is cubic and the values for $\lambda_{p}$ can be found with the aid of Cardano's formula. Note the homogeneity of $\lambda_{p}: \lambda_{p}(s \hat{\xi})=s \lambda_{p}(\hat{\xi})$, which also implies that $-\lambda_{p}(-\hat{\xi})=\lambda_{p}(\hat{\xi})$.

The $\alpha^{(p)}$ follow from the initial conditions (cf. (2.10)),

$$
\sum_{p<0, p>0}\left(-\lambda_{p}\right)^{\prime} \alpha^{(p)} e_{m}^{(p)}=\tilde{\phi}_{m k}^{(\prime)} a_{k}, \ell=0, \ldots, 2 n-1,
$$

where $\tilde{\phi}_{m k}^{(/)}$follows from $\phi_{m k}^{(/)}$by replacing $\hat{o}_{r}$ with $\hat{\xi}_{r}$, employing Gel'fand's formula. The resulting equations reduce to

$$
\begin{gathered}
\sum_{p<0, p>0} \alpha^{(p)} e_{m}^{(p)}=0, \\
-\sum_{p<0, p>0}\left(e_{m}^{(p)} \hat{i}_{p}\right) \alpha^{(p)}=a_{m} .
\end{gathered}
$$

In view of the $\pm p$ symmetry, the first equation implies

$$
\alpha^{(-p)}=-\alpha^{(p)} ;
$$

then the second equation implies

$$
\alpha^{(p)}=-\frac{1}{2 \lambda_{p}}\left(e_{k}^{(p)} a_{k}\right) .
$$

The weighting functions $\alpha^{(p)} e_{m}^{(p)}=-a_{k}\left(2 \lambda_{p}\right)^{-1}\left(e_{k}^{(p)} e_{m}^{(p)}\right)$ lead to the well-known dyadic form of the Green's tensor. Using that the distribution $\operatorname{Re}\left\{i^{n-1} \chi_{-n+1}\left(\hat{\xi}_{j} x_{j} \pm\right.\right.$ $\left.\left.\lambda_{p}(\hat{\xi}) t-i 0\right)\right\}$ is even in $\hat{\xi}_{j} x_{j} \pm \lambda_{p}(\hat{\xi}) t$, and that $\hat{\xi}_{j} x_{j} \pm \lambda_{p}(\hat{\xi}) t$ is odd in $\hat{\xi}$ (point mirror symmetry) the expression in (2.34) can be reduced to a sum over positive values of $p$ 's and integrals, over the hemisphere of $\Omega$. 
Using the symmetry in time again, the plane-wave expansion of the scalar function $E$ is found to be

$$
\begin{aligned}
E= & -\frac{i^{n-1}}{2^{n} \pi^{n-1}} \times \sum_{p>0} \int_{\Omega} A^{(p)}(\hat{\zeta})\left[\chi_{n-1}\left(\hat{\zeta}_{j} x_{j}-\lambda_{p}(\hat{\zeta}) t\right)\right. \\
& \left.-\chi_{n-1}\left(\hat{\zeta}_{j} x_{j}+\lambda_{p}(\hat{\xi}) t\right)\right] \mathrm{d} S(\hat{\zeta}) .
\end{aligned}
$$

The initial conditions for $E$ now lead to

$$
2 \sum_{p>0}\left(-\lambda_{p}\right)^{2 / 0^{-1}} A^{(p)}=\delta_{2 / 0-1,2 n-1}, \ell_{0}=1, \ldots, n .
$$

Since (2.38) implies

$$
a\left(\hat{\xi},-i_{i}\right)=\prod_{p>0}\left(\hat{i}^{2}-i_{p}^{2}(\hat{\xi})\right)
$$

the solution of $(2.46)$ is given by

$$
A^{(p)}=-\frac{1}{\left.\left(\hat{o}_{\lambda} a\right)\right|_{\lambda=\lambda, p}} .
$$

Using the homogeneity of $a$, viz., $a(s \hat{\xi},-s \hat{\lambda})=s^{2 n} a(\hat{\xi},-\hat{i})$, it follows that

$$
\left.\left(-\lambda \hat{\partial}_{\lambda} a\right)\right|_{\lambda=\lambda_{p}}=\left.\left(\hat{\xi}_{i} \hat{\partial}_{\hat{\xi}_{i}} a\right)\right|_{\lambda=\lambda_{p}}
$$

and (2.48) can be written as

$$
A^{(p)}=\frac{\lambda_{p}}{\left.\left(\hat{\xi}_{i} \hat{\xi}_{\hat{\xi}_{i}} a\right)\right|_{\lambda=\lambda_{p}}} .
$$

\section{The Herglotz-Petrovsky-Leray Formulae}

The Geometry of the Slowness and the Group Velocity Hypersurfaces. In this subsection we summarize the basic properties of the varieties which will play a rôle in the further analysis. For a detailed discussion we refer the reader to Duff [15], Musgrave [32], and Payton [36].

Let the slowness cone $\hat{A} \subset \hat{Z}$ be defined through

$$
\hat{A}: a=0 \text {. }
$$

The slowness or ray vector $\xi$ is introduced as

$$
\hat{\xi}_{j}=\lambda_{p} \xi_{j}, p=1, \ldots, n \text {. }
$$

If $\hat{\xi} \in \Omega$ then $\|\xi\|=1 /\left|\lambda_{p}\right|$ equals the phase slowness. In fact, (3.2) represents the transition from $\hat{Z} \simeq \mathbb{C}^{n+1}$ to the projective space $^{1} Z \simeq \mathbb{C} \mathbb{P}^{n}$. Let the function $H$ be given by

$$
H\left(\xi_{1}, \ldots, \xi_{n}\right)=a\left(\xi_{1}, \ldots, \xi_{n},-1\right)
$$

\footnotetext{
${ }^{1}$ Un-hatted variables are projective variables throughout.
} 
(Note that $H$ is not homogeneous in $\xi_{1}, \ldots, \xi_{n}$.) Then the slowness hypersurface is defined by

$$
A: H=0
$$

(this equals the intersection of the slowness cone $\hat{A}$ with the plane $\lambda_{0}=1$ ). In any local cone (with its vertex at the origin) the entirely real solution of the latter equation, $\operatorname{Re}\{A\}$, consists of $n$ sheets. Every sheet corresponds with a (double) mode $( \pm) p$ and can be covered with (two) almost everywhere holomorphic coordinate patches.

In general, the sheets can have multiple points, where two sheets may even be tangent [14]. In the non-generic case (requiring a certain (rotational) symmetry in the stiffness) sheets can have curves or higher-dimensional surfaces of multiple points (where two sheets are tangent or where two sheets intersect). In the latter case the space-time singularities are of a different nature: only a hypersurface of multiple points of codimension 2 leads to an additional arrival. There the coordinate patches cannot be holomorphic. (For an analysis of the local slowness surface parametrization near a conical point in three dimensions, see Musgrave [33].) In geophysical applications, where one investigates finite frequency representations of the fundamental solution, "almost" (i.e., perturbed) intersecting sheets or tangent sheets have to be treated with care: they lead to quasi-singularities (viz., the "pinch" singularity in case of "almost" intersecting and the "kissing" singularity in case of tangent sheets) in the sense that the coupling between the modes have to be taken into account.

If points of tangency occur, the slowness surface as a whole is called singular; if the sheets are entirely disconnected, the surface is said to be regular. Each sheet is smooth and either locally convex or concave (elliptic points) or locally (Morse) saddle shaped (hyperbolic points) except at points or curves where (one of) the principal curvatures vanish (parabolic points, e.g., inflection points in a plane through the origin of the slowness space). In this paper we will focus on the latter singularities.

The group velocity $\left(v^{\mathrm{gr}}\right)$ hypersurface is known to be the polar reciprocal of $\operatorname{Re}\{A\}$ through the transform

$$
\xi_{j} \frac{x_{j}}{t}=1
$$

When $\xi_{j} \in \operatorname{Re}\{A\}$ varies, the $\left\{x_{i}, t\right\}$ span the variety $W \subset \hat{Z}^{\prime}$. Another way of introducing this so-called characteristic surface, spanned by the rays associated with the wave operator, is the following:

$$
W: S(x, t)=0
$$

is the surface in space-time $\hat{Z}^{\prime}$ following from the eikonal equation

$$
a\left(\hat{o}_{i} S, \hat{o}_{t} S\right)=0 \text {. }
$$

The relevant class of characteristic surfaces is formed by the (conical) surfaces with their vertices at the origin (the location of the source for the Green's tensor). If $\partial_{t} S=(-) 1, S$ is the travel time, being the integral of group slowness along the ray. Then $H\left(\partial_{i} S\right)=0$ and $H$ is the Hamiltonian of the ray system of equations. In inhomogeneous media $H$ is defined through the principal symbol of the wave operator. Later on, for notational convenience, we will replace $S$ by $F$. 
At $t=1$, i.e., in the projective coordinates $\left\{x_{j} / t\right\}$, the characteristic surface reduces to the wave front $\Sigma_{w} \subset Z^{\prime}$. In the projective coordinates $\left\{x_{1} / x_{n}, \ldots, x_{n-1} / x_{n}\right.$, $\left.t / x_{n}\right\}$ we obtain the so-called diffraction surface $\Sigma_{d}$. The singularities of the wave front and the diffraction surface are similar, i.e., $t$ and $x_{n}$ play equivalent rôles, hence we can loosely consolidate the notation to $\Sigma$. The wave front coincides with the group velocity surface. The surfaces $\Sigma_{d}$ and $\operatorname{Re}\{A\}$ are called each other's dual. This terminology will be explained in Sect. 4. It is found that

$$
\frac{x_{j}^{(p)}}{t}=\frac{\hat{o}_{\hat{\xi}_{j}} i_{p}^{2}}{\hat{\zeta}_{i} \hat{\sigma}_{\hat{\xi}_{i}} \lambda_{p}^{2}} \text { or } \frac{x_{j}}{t}=\frac{\partial_{\xi_{j}} H}{\xi_{i} \partial_{\xi_{i}} H}=v_{j}^{\mathrm{gr}} .
$$

Using (2.36), the latter representation simplifies to

$$
\frac{x_{i}^{(p)}}{t}=\frac{e_{k}^{(p)} C_{k m}^{r s} e_{m}^{(p)} \hat{\xi}_{s}}{\hat{\xi}_{r^{\prime}} e_{k^{\prime}}^{(p)} C_{k^{\prime} m^{\prime}}^{r^{\prime} s^{\prime}} e_{m^{\prime}}^{(p)} \hat{\xi}_{s^{\prime}}}=e_{k}^{(p)} C_{k m}^{r s} e_{m}^{(p)} \xi_{s} .
$$

The degree of the wave front or group velocity surface cannot exceed the so-called class number of the slowness surface. The class number of the degree $D=2 n$ surface in $n$ dimensions is given by $D(D-1)^{n-1}$, and counts the total number of linearly independent tangents that can be drawn on a closed surface. Since in the 3-dimensional problem the degree of the slowness surface is 6 , the degree of $\Sigma_{w}$ is at most 150 [15]. As $\operatorname{Re}\{A\}$ and hence $\Sigma_{w}$ are point symmetric in the origin, a half line from the origin in space will meet $\Sigma_{w}$ at most in 75 points. In practice, the degree will be less than 150 (see e.g. Salmon [40] and Duff [15]). Still the degree of $\Sigma_{w}$ can be formidable, which generally prevents one from writing down an equation for $\Sigma_{w}$ in closed form (see also Musgrave [32]).

Now, the globally innermost slowness sheet of the slowness surface (which need not to be smooth) must always be locally strictly convex. Thus, if the inner sheet does not have any multiple points with any other sheet, the fastest characteristics simply span a smooth convex surface $\hat{\partial} \hat{K}$, say. However, if the innermost sheet does contain multiple points, its normal will pass discontinuities. Such a discontinuity corresponds to a discontinuity of position, but the normal to the associated wave front will be continuous. As the tangent plane to the innermost sheet turns about the multiple point, the dual point on the wave front moves along a given multiple $(n-1)$-dimensional plane surface ("lid"). (Double curves on the innermost slowness sheet correspond to portions of ruled surfaces in space). Thus, the convex completion of the variety of fastest characteristics is obtained. The latter forms the convex hull, which again will be denoted as $\partial \hat{K}$. $\partial \hat{K}$ is the boundary of the variety $\hat{K} \subset \hat{Z}^{\prime}$ of all characteristic surfaces. The convex hull is an algebraic surface known as the wave cone. Its physical significance resides in the fact that it limits the region of influence of a disturbance originating at the origin. In fact, $\partial \hat{K}$ is the boundary of the smallest convex region containing all $n$ sheets of $W$. A property of the solution to be discussed next is that it is singular on $W$ (the geometrical arrivals). Thus,

$$
\operatorname{supp} E \subset \hat{K}, \quad \operatorname{sing} \operatorname{supp} E \subset W .
$$

The singularities on $\operatorname{Re}\{A\}$ are dual to the singularities on $\Sigma_{d}$. To a double point on $\operatorname{Re}\{A\}$ corresponds a double tangent on $\Sigma_{w}$. To a curve of inflections on $\operatorname{Re}\{A\}$ corresponds a cuspidal edge on $\Sigma_{w}$; such a curve of vanishing principal curvature can be very complicated, see also Every [17]. From a mathematical point of view 
SLOWNESS

Vertical Cross-section

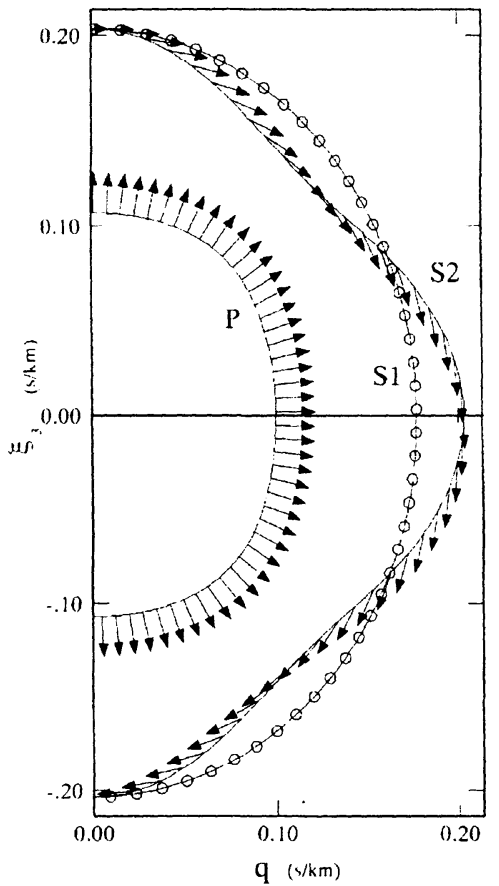

VELOCITY

Vertical Cross-section

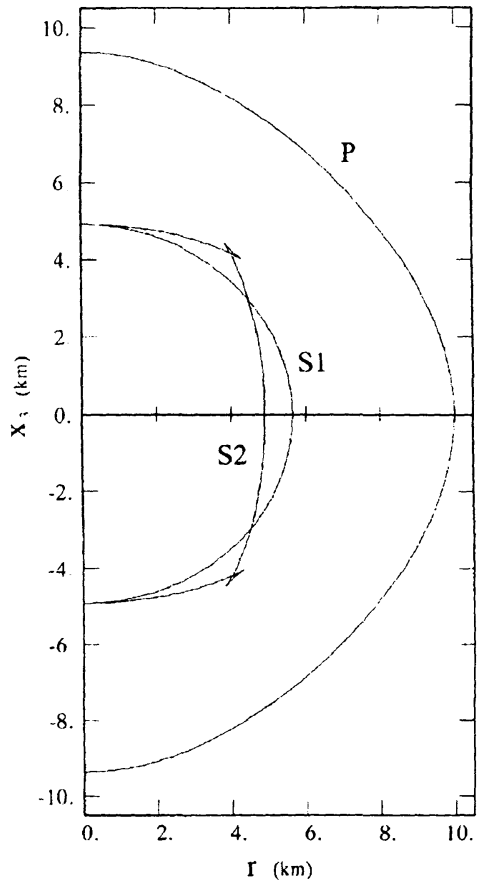

Fig. 3.1. The slowness surface and wave front of a hexagonal medium (an example of a cusp)

the slowness surface is therefore easier to manage. In fact, as we shall now discuss, the fundamental solution to the wave equation can be recast in terms of an integral over this slowness surface. The solution, in particular near the wave front, is mostly sensitive to the topology of the slowness surface close to the singular points. We will extract the topological part of the solution by studying more closely the effect of singular points on the integral representation of the fundamental solution.

To illustrate the geometrical features discussed so far, we consider two realistic media in three dimensions: a particular hexagonal one with circles of vanishing principal curvature leading to a cusp on the wave front, and a particular orthorhombic one, which is a perturbation of the hexagonal one, with swallowtail singularities. The real slowness surface, the wave front and the corresponding polarisation vectors are evaluated. Let the vertical axis be a principal axis of symmetry. Fig. 3.1 shows a vertical section of the hexagonal medium; Figs. 3.2-3.3 show vertical sections at different azimuths of the particular orthorhombic medium.

Integration over the Real Slowness Surface. The sum over the modes combined with the integral over the unit sphere can now be expressed as an integral over the real slowness surface (using local coordinates in a small cone and the outward 
SLOWNESS

Vertical Cross-section

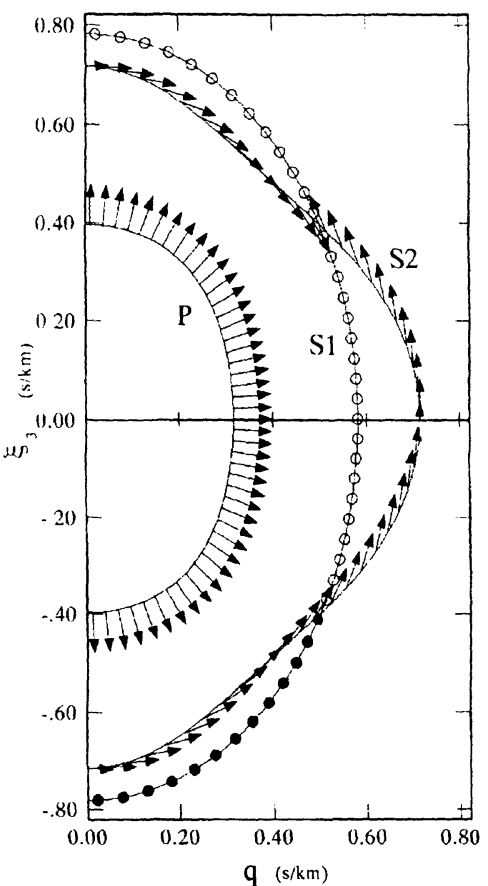

VELOCITY

Vertical C'ross-section

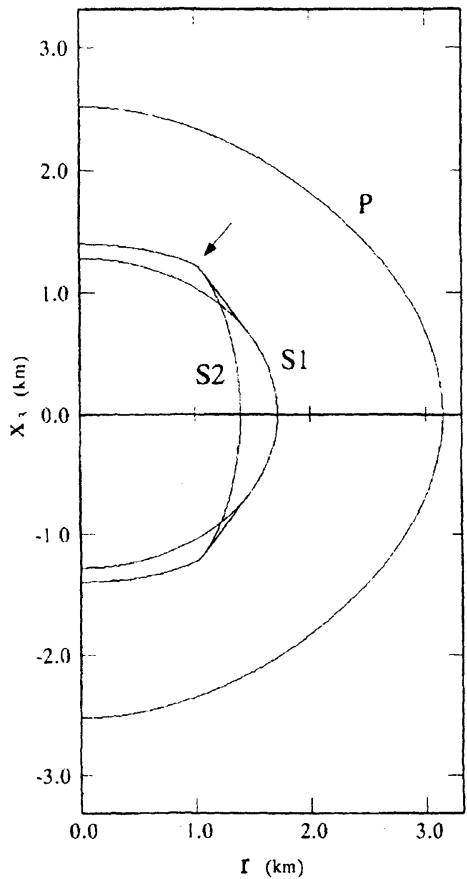

Fig. 3.2. The slowness surface and wave front of an orthorhombic medium at zero degrees (an example of an intersection of swallowtail, indicated by the arrow in the figure on the right)

normal or group direction $\left.\partial_{\xi} H /\left\|\partial_{\xi} H\right\|\right)$. For a discussion see also Auld [9] or John [25]. Let the volume form on $\operatorname{Re}\{A\}$ be denoted as $\mathrm{d} S(\xi)$. Then (see Fig. 3.4)

$$
\mathrm{d} S(\xi)=\frac{\|\xi\|^{n-1}}{|\cos \theta|} \mathrm{d} S(\hat{\xi})
$$

where 0 is the angle between the group velocity and phase slowness vectors. Hence

$$
\mathrm{d} S(\hat{\xi})=\frac{\left|\xi_{j} \partial_{\xi_{j}} H\right|}{\|\xi\|^{n}\left\|\partial_{\xi} H\right\|} \mathrm{d} S(\xi) .
$$

Further, the weighting function $A^{(p)}$ transforms as (cf. (2.49) and we extracted the positive phase velocities $p>0$ )

$$
A^{(p)} \rightarrow \frac{\|\xi\|^{2 n-1}}{\xi_{j} \partial_{\xi_{j}} H},
$$

while (cf. (2.21))

$$
\chi=-1\left(\hat{\xi}_{j} x_{j}-\lambda_{p}(\hat{\xi}) t\right) \rightarrow\|\xi\|^{-z+1} \chi_{z-1}\left(\xi_{j} x_{j}-t\right) .
$$


SLOWNESS

Vertical Cross-section

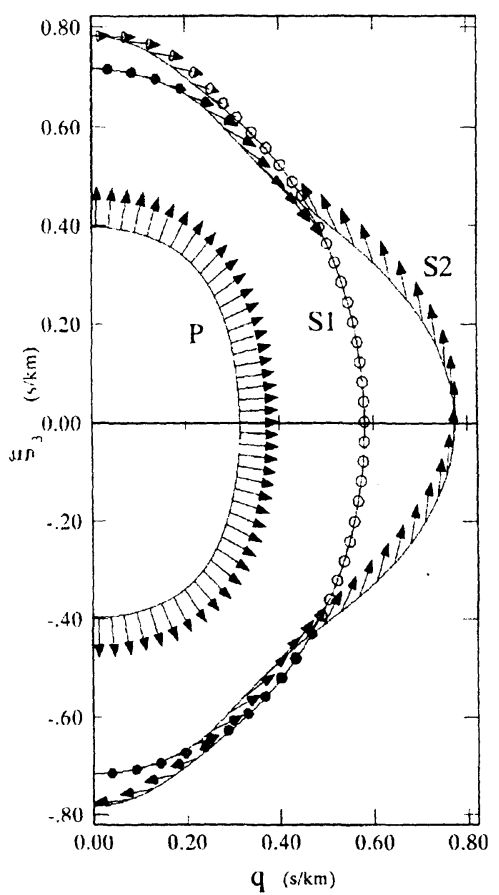

VELOCITY

Vertical Cross-section

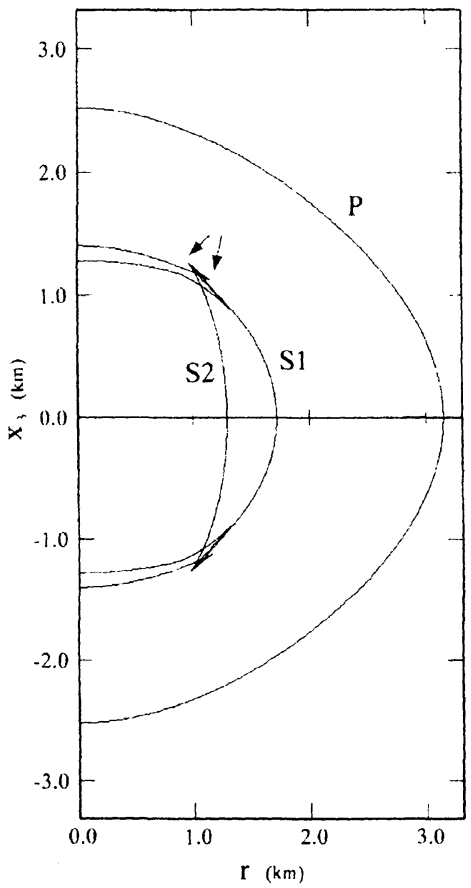

Fig. 3.3. The slowness surface and wave front of the orthorhombic medium of the previous figure at 70 degrees

Set

$$
\mathrm{d} \sigma(\xi)=\frac{\mathrm{d} S(\xi)}{\left\|\hat{\partial}_{\xi} H\right\|} \operatorname{sgn}\left(\xi_{j} \partial_{\xi_{j}} H\right) ;
$$

upon substituting the latter results in (2.45) and taking the limit $z \rightarrow n$, we obtain

$$
E=\frac{-i^{n-1}}{2^{n} \pi^{n-1}} \begin{cases}\operatorname{Re}\left\{\int_{\operatorname{Re}\{A\}}\left[\chi_{n-1}\left(\xi_{j} x_{j}-t\right)-\chi_{n-1}\left(\xi_{j} x_{j}+t\right)\right] \mathrm{d} \sigma(\xi)\right\} & \text { if } n \text { is odd } \\ i \operatorname{Im}\left\{\int_{\operatorname{Re}\{A\}}\left[\chi_{n-1}\left(\xi_{j} x_{j}-t\right)-\chi_{n-1}\left(\xi_{j} x_{j}+t\right)\right] \mathrm{d} \sigma(\xi)\right\} & \text { if } n \text { is even. }\end{cases}
$$

This formula, known as the HPL formula, implies that the fundamental solution can be expressed in terms of so-called non-evanescent constituents only. Here, we made use of the fact that the system is non-dispersive. Note that the imaginary part involves a logarithm, while the real part does not. Also note that the full solution is found this way, which does not contain any divergencies at the singularities of the wave fronts. Furthermore, $\operatorname{Re}\left\{i^{n-1} \chi_{n-1}\left(\xi_{j} x_{j} \pm t-i 0\right)\right\}$ is even in $\xi_{j} x_{j} \pm t$. Hence, (3.16) may be written as 


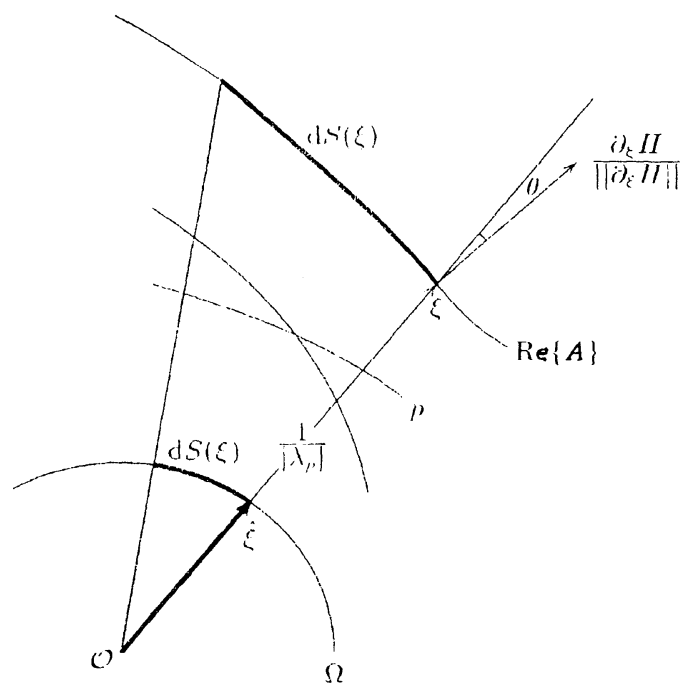

Fig. 3.4. The projection of the unit sphere onto the slowness surface

$$
E=\frac{-i^{n-1}}{2^{n} \pi^{n-1}} \begin{cases}\operatorname{Re}\left\{\int_{\operatorname{Re}\{A\}}\left[\chi_{n-1}\left(t-\xi_{j} x_{j}\right)-\chi_{n-1}\left(t+\xi_{j} x_{j}\right)\right] \mathrm{d} \sigma(\xi)\right\} & \text { if } n \text { is odd } \\ i \operatorname{Im}\left\{\int_{\operatorname{Re}\{A\}}\left[\chi_{n-1}\left(t-\xi_{j} x_{j}\right)-\chi_{n-1}\left(t+\xi_{j} x_{j}\right)\right] \mathrm{d} \sigma(\xi)\right\} & \text { if } n \text { is even }\end{cases}
$$

We exploit the fact that the integrand is even in $t \pm \xi_{j} x_{j}$ further. It has been observed that $\operatorname{Re}\{A\}$ is invariant under the point reflection in the origin in $\xi$-space. Now, choose a direction of preference, $x_{n}$ say, relative to the principal axes of symmetry of the medium. We can separate the "ups" and "downs" with respect to the preferred direction either according to the phase ("ph") direction of propagation, viz. $\xi_{n}<0$ or $>0$, or according to the group ("gr") direction of propagation, viz. $\partial_{\xi_{n}} H<0$ or $>0$. We set $\operatorname{Re}\{A\}=A_{+}^{\mathrm{ph}} \cup A_{-}^{\mathrm{ph}}$ or $\operatorname{Re}\{A\}=A_{+}^{\mathrm{gr}} \cup A_{-}^{\mathrm{gr}}$. Then $A_{+}^{\mathrm{gr}}$ corresponds with the upgoing waves, identified by the wave front $\Sigma_{w}=\Sigma_{+} \cup \Sigma_{-}$ through the polar reciprocal of $A_{+}^{\mathrm{gr}} \cup A_{-}^{\mathrm{gr}}$. According to the statement below (2.44) we can always write

$$
E=-\frac{i^{n-1}}{2^{n-1} \pi^{n-1}} \int_{A_{+}}\left[\chi_{n-1}\left(t-\xi_{j} x_{j}\right)-\chi_{n-1}\left(t+\xi_{j} x_{j}\right)\right] \mathrm{d} \sigma(\xi) .
$$

This way we have introduced an orientation for $\operatorname{Re}\{A\}$. Now choose coordinates on $A_{+}$. For this purpose consider the orthogonal projection $\mathscr{C}$ of $A_{+}$on the plane $\left\{\xi_{n}=0\right\}$ and let $\left(\xi_{1}, \ldots, \xi_{n-1}\right)$ be the coordinates. The parametric representation of the slowness surface $A_{+}$is then given by $\left\{\xi_{1}, \ldots, \xi_{n-1}, f\left(\xi_{1}, \ldots, \xi_{n-1}\right)\right\}$, where $f$ represents a $n$-plet. Then

$$
H=\prod_{|p|=1}^{n}\left[\xi_{n}-f_{p}\left(\xi_{1}, \ldots, \xi_{n-1}\right)\right] .
$$

This equality allows us to derive $H$ from $f$, which will be needed in the next section. Later on, the direction of preference will be chosen in the direction of observation. At the singular points contributing to this direction of propagation, a 
principal curvature or its derivative may vanish. Thus, in the preferred coordinate system, the occurrence of a singular point on a particular sheet corresponds with multiple $(\mu \geqq 1)$ roots of the equation

$$
\hat{o}_{\xi_{1}, \ldots, \xi_{n-1}} f=0 .
$$

At these roots the normal to the slowness surface, i.e., the group direction, must be parallel to the $\tilde{\xi}_{n}$-axis, which in turn translates into the condition

$$
\hat{\partial}_{\xi_{1}, \ldots, \xi_{n-1}} H=0 \text { with } H=0 \text {. }
$$

We have (see Fig. 3.4)

$$
\frac{\mathrm{d} S(\zeta)}{\left\|\hat{\partial}_{\xi} H\right\|}=\frac{d \xi_{1} \ldots d \xi_{n-1}}{\left|\hat{\partial}_{\xi_{n}} H\right|},
$$

and hence, restricting to $A_{+}^{\mathrm{gr}}$,

$$
\mathrm{d} \sigma(\xi) \rightarrow \frac{d \xi_{1} \ldots d \xi_{n-1}}{\partial_{\xi_{n}} H} .
$$

Note that this $(n-1)$-form has poles at the branch points of our parametrization $f$. We will omit the superscript "gr" in the further analysis.

Cagniard Green's Function. At this stage, to reduce the integral, $E$ is written as

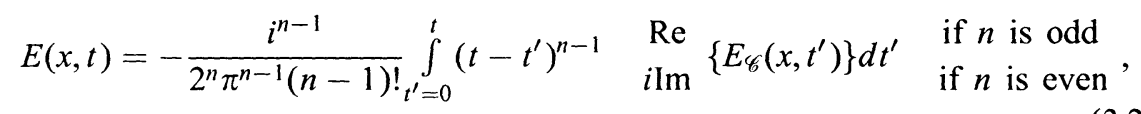

which means that

$$
\hat{o}_{t}^{n-1} E(x, t)=-\frac{i^{n-1}}{2^{n} \pi^{n-1}} \int_{t^{\prime}=0}^{t} E_{\mathscr{L}}\left(x, t^{\prime}\right) d t^{\prime} .
$$

Here, $E_{\mathscr{C}}$ is denoted as the Cagniard Green's function and is given by

$$
E_{\mathscr{C}}\left(x, t^{\prime}\right)=2 \int_{A_{+}^{\mathrm{gr}}}\left[\Delta\left(t^{\prime}-\xi_{j} x_{j}-i 0\right)-\Delta\left(t^{\prime}+\xi_{j} x_{j}-i 0\right)\right] \mathrm{d} \sigma(\xi) .
$$

We will now redefine $E$, which will be the only form of the solution we will consider in the further analysis:

$$
E(x, t) \equiv \int_{t^{\prime}=0}^{t} E_{\mathscr{C}}\left(x, t^{\prime}\right) d t^{\prime} .
$$

It is observed that integrals of the type (3.25) have integrands which are strictly rational in the slowness variables (in this respect note that $\partial_{\xi_{n}} H$ is polynomial).

Petrovsky Cycles. We consider the decomposition $\operatorname{Re}\{A\}=A_{+}^{\mathrm{ph}} \cup A_{-}^{\mathrm{ph}}$. Employing the theorem of residues of complex function theory, one of the integrals in the horizontal slowness variables can be evaluated. To this end, we first will choose alternative coordinates on the real slowness surface $A_{+}^{\mathrm{ph}}$, viz. the ones through the projection: 


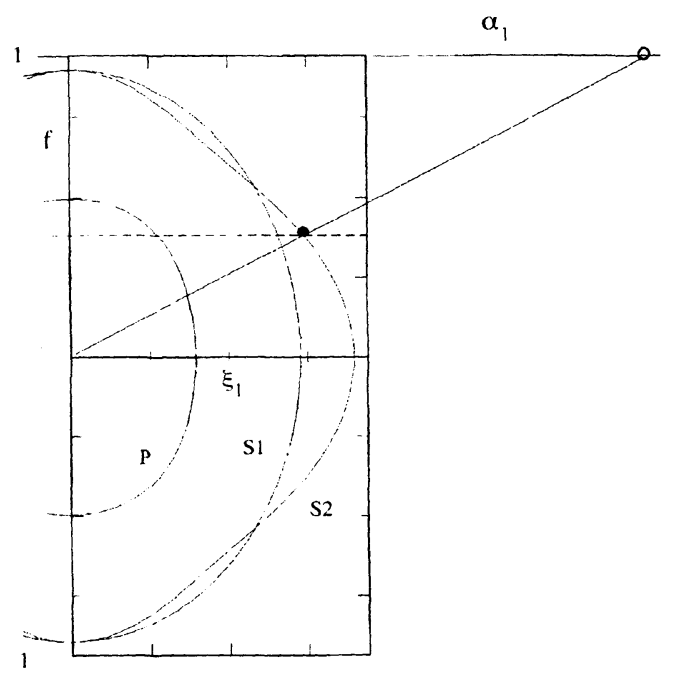

Fig. 3.5. A particular chart on the real slowness surface

$$
\xi_{1}=f \alpha_{1}, \ldots, \quad \xi_{n-1}=f \alpha_{n-1}, \quad \xi_{n}=f, \quad \text { where } \alpha_{1}, \ldots, \alpha_{n-1} \in \mathbb{R}
$$

(see Fig 3.5). Such a change of variables is also discussed in [18], p. 81. Now set

$$
H=H\left(f \alpha_{1}, \ldots, f \alpha_{n-1}, f\right) \equiv H(f) .
$$

Using the coordinates introduced in (3.27), we set

$$
\xi_{j} x_{j}=f \mathscr{X} \text { with } \mathscr{X}=\alpha_{j} x_{j}, \alpha_{n} \equiv 1 .
$$

According to the point mirror symmetry of $\operatorname{Re}\{A\}$, we label the roots $f_{p}$ of the equation $H(f)=0$ as $p= \pm 1, \ldots, \pm n$. Then

$$
\int_{A_{ \pm}^{\mathrm{ph}}} \cdots \frac{d \xi_{1} \cdots d \xi_{n-1}}{\left(\partial_{\xi_{n}} H\right)(\xi)} \rightarrow \int_{\alpha \in \mathbb{R}^{n-1} \pm p=1} \sum_{p=1}^{n} \cdots \frac{\left( \pm f_{p}\right)^{n-1} d \alpha_{1} \cdots d \alpha_{n-1}}{\left( \pm \mathrm{d}_{f} H\right)\left(f_{p}\right)} .
$$

To simplify the right-hand side of (3.30), we consider Euler's formula: let $P(\tau)$ be a polynomial in $\tau$ of degree $D_{P}$ and let $Q(\tau)$ be a polynomial in $\tau$ of degree $D_{Q}$. Assuming that $D_{P}<D_{Q}-1$, the formula states that

$$
\sum_{\tau=\tau} \frac{P(\tau)}{\text { (roots of } Q)}=0 .
$$

Combining the "ups" and "downs" in $E_{\mathscr{C}}$ and applying Euler's formula to the polynomials $P(f)=f^{n-1}, Q(f)=(t \pm \mathscr{X} f-i 0) H(f)$ (the expression for $\Delta$ being substituted into $(3.30)$ ) yields

$$
\sum_{ \pm p=1}^{n} \frac{f_{p}^{n-1}}{\left(t \pm \mathscr{X} f_{p}-i 0\right)\left(\mathrm{d}_{f} H\right)\left(f_{p}\right)}=-\left(\mp \frac{t-i 0}{\mathscr{X}}\right)^{n-1} \frac{1}{( \pm \mathscr{X}) H(\mp(t-i 0) / \mathscr{X}}
$$


We arrive at the following expression for the Cagniard's Green's function:

$$
\begin{aligned}
E_{\mathscr{C}}(x, t)= & \frac{i}{\pi_{x \in \mathbb{R}^{n-1}}}\left[\left(\frac{t-i 0}{\mathscr{X}}\right)^{n-1} \frac{1}{\mathscr{X} H((t-i 0) / \mathscr{X})}\right. \\
& \left.+\left(-\frac{t-i 0}{\mathscr{X}}\right)^{n-1} \frac{1}{\mathscr{X} H(-(t-i 0) / \mathscr{X})}\right] d \alpha_{1} \cdots d x_{n-1} .
\end{aligned}
$$

We extract the integral over $\alpha_{n-1}$ and note that the contour of integration (the real axis) can be closed both in the upper and the lower complex half plane with vanishing contributions at infinity. Label the simple poles $\alpha_{n-1}\left(\alpha_{1}, \ldots, \alpha_{n-2}, x, t\right)$ of the integrands, arising from the zero's of $H( \pm(t-i 0) / \mathscr{X})$, as $\alpha_{n-1}^{(p)}$. Introduce the polynomials $G_{\mp}$ in $\alpha_{1}, \ldots, \alpha_{n-1}$ as

$$
G_{\mp}=\mathscr{X}^{D} H(\mp(t-i 0) / \mathscr{X}) \text {. }
$$

Then

$\operatorname{Res}_{\alpha_{n-1}^{(p)}}\left[\left(\frac{t-i 0}{\mathscr{X}}\right)^{n-1} \frac{1}{\mathscr{X} H(\mp(t-i 0) / \mathscr{X})}\right]=\operatorname{Res}_{\alpha_{n-1}^{(p)}}\left[(t-i 0)^{n-1} \frac{\mathscr{X}^{n}}{G_{\mp}\left(\alpha_{1}, \ldots, \alpha_{n-1}, x, t\right)}\right]$.

Without restriction, we can assume that the direction of observation coincides with the $x_{n}$-axis. This implies that everywhere $\xi_{j} x_{j}$ (or $\mathscr{X}$ ) can be replaced by $\xi_{n} x_{n}$ (or $x_{n}$ ). The new coordinates are denoted as the old ones. After the rotation (i.e., $\left.x_{1}=\cdots=x_{n-1}=0\right)(3.33)$ reduces to

$$
\begin{aligned}
& \operatorname{Res}_{\alpha_{n-1}^{(p)}}\left[(t-i 0)^{n-1} \frac{x_{n}^{n}}{G \mp\left(\alpha_{1}, \ldots, \alpha_{n-1}, x_{n}, t\right)}\right] \\
& =(t-i 0)^{n-1}\left(\frac{x_{n}^{n}}{\left.\left(\partial_{\alpha_{n-1}} G_{\mp}\right)\right|_{\alpha_{n-1}^{(p)}}}\right) .
\end{aligned}
$$

Using $\xi_{j}=\left(t / x_{n}\right) \alpha_{j}$ and $\xi_{n-1}^{(p)}=\left(t / x_{n}\right) \alpha_{n-1}^{(p)}$, this expression transforms back to

$$
\begin{aligned}
& (t-i 0)^{n-1}\left(\frac{x_{n}^{n}}{\left.\left(\partial_{\alpha_{n-1}} G_{\mp}\right)\right|_{\alpha_{n-1}^{(p)}}}\right) d \alpha_{1} \cdots d \alpha_{n-2} \\
& \quad \rightarrow \frac{1}{x_{n}} \frac{1}{\left(\partial_{\xi_{n-1}} H\right)\left(\xi_{1}, \ldots, \xi_{n-2}, \xi_{n-1}^{(p)}, \mp\left(t / x_{n}\right)\right)} d \xi_{1} \cdots d \xi_{n-2} .
\end{aligned}
$$

The time axis now coincides with the $\xi_{n}$-axis (see Fig. 3.4) hence (see (3.26))

$$
\frac{1}{x_{n}} d t^{\prime} \rightarrow d \xi_{n}
$$

Note that the poles parametrize the intersection of $A$ with the hyperplane $X_{t}$ defined by

$$
X_{t}: \mp \xi_{j} x_{j}-t=\mp f \mathscr{X}-t=\mp f x_{n}-t=0 .
$$


The poles are either real-valued or occur in complex conjugated pairs, depending on the time $t$. Near the arrival times of the wave constituents complex and real poles will come together. Thus

$$
\begin{aligned}
& \int_{\alpha_{n-1} \in \mathbb{R}} \frac{1}{\mathscr{X}}\left[\left(\frac{t-i 0}{\mathscr{X}}\right)^{n-1} \frac{1}{H((t-i 0) / \mathscr{X})}+\left(-\frac{t-i 0}{\mathscr{X}}\right)^{n-1} \frac{1}{H(-(t-i 0) / \mathscr{X})}\right] d \alpha_{n-1} \\
& \quad=2 \pi i\left(\sum^{\text {upper }} \text { residues }\right)=-2 \pi i\left(\sum^{\text {lower }} \text { residues }\right) \\
& =\pi i\left(\sum^{\text {upper }} \text { residues }-\sum^{\text {lower }} \text { residues }\right)
\end{aligned}
$$

where $\sum^{\text {upper }}$ refers to the sum over poles in the upper complex half plane and $\sum^{\text {lower }}$ refers to the sum over poles in the lower complex half plane. Taking into account the term $-i 0$ in the arguments of the integrand, we also distinguish between "real" poles in the upper and lower complex half planes. Thus

$$
\begin{aligned}
\sum^{\text {upper }} \text { residues }-\sum^{\text {lower }} \text { residues }= & \sum_{\text {complex poles }}^{\text {upper }} \text { residues }-\sum_{\text {complex poles }}^{\text {lower }} \text { residues } \\
& +\sum_{\text {real poles }}^{\text {upper }} \text { residues }-\sum_{\text {real poles }}^{\text {lower }} \text { residues . }
\end{aligned}
$$

Since the complex poles occur in conjugated pairs, the total sum over the complex poles is purely imaginary. Naturally the total sum over the real poles is real. Thus, for odd $n$ we get the contributions from the real poles only; they define the real Petrovsky cycles. For even $n$ we get the contributions from the complex poles only; they define the complex Petrovsky cycles. The cycles are denoted by $\hat{\gamma} \gamma$.

Cagniard-De Hoop Contours. Reconsider the poles of the previous subsection. To find the poles, in principle, the equation

$$
f\left(\xi_{1}, \ldots, \xi_{n-1}\right) x_{n}= \pm(t-i 0)
$$

must be solved. Here, time $t$ is real, but also $\xi_{1}, \ldots, \xi_{n-2}$ are kept real. It $t$ is sufficiently small, the (pole) solution for $\xi_{n-1}$ is naturally real. However, if $t$ is large, $\breve{\zeta}_{n-1}$ must become complex. To relate these poles to the Cagniard-De Hoop contours, we introduce the polar coordinates

$$
\begin{aligned}
\xi_{n-1} & =q \sin \left(\psi_{n-2}\right), \xi_{n-2}=q \cos \left(\psi_{n-2}\right) \sin \left(\psi_{n-3}\right), \\
\xi_{n-3} & =q \cos \left(\psi_{n-2}\right) \cos \left(\psi_{n-3}\right) \sin \left(\psi_{n-4}\right), \ldots, \\
\xi_{2} & =q \cos \left(\psi_{n-2}\right) \cdots \cos \left(\psi_{2}\right) \sin \left(\psi_{1}\right), \\
\xi_{1} & =q \cos \left(\psi_{n-2}\right) \cdots \cos \left(\psi_{2}\right) \cos \left(\psi_{1}\right),
\end{aligned}
$$

with $\psi_{1} \in[0,2 \pi)$ and $\psi_{2}, \ldots, \psi_{n-2} \in[0, \pi)$. We will use the shorthand notation $\psi=\left(\psi_{1}, \ldots, \psi_{n-2}\right)$ and incorporate the Jacobian, given by

$$
q^{n-2} \sin ^{n-3}\left(\psi_{n-2}\right) \sin ^{n-4}\left(\psi_{n-3} \cdots \sin \left(\psi_{2}\right) d q d \psi,\right.
$$

through $\mathrm{d} \sigma(\xi) \rightarrow \mathrm{d} \sigma(q, \psi)$. 
Rather than applying the residual theorem to the $\alpha_{n-1}$-integral, we now apply the same theorem to the $q$-integral. If we also introduce polar coordinates $(r, \phi)$ in the $\left(x_{1}, \ldots, x_{n-1}\right)$-plane, the poles in the complex $q$-plane are the solutions of the equation

$$
q r \cos (\eta)+f(q, \psi) x_{n}= \pm(t-i 0) \quad \text { or } \quad f(q, \psi) x_{n}= \pm(t-i 0)
$$

upon rotation of the spatial coordinates, where $\eta$ can be expressed in the angles $\psi$ and $\phi$.

The solution of either (3.39) or (3.41) can be interpreted as the intersection of the hyperplane

$$
X_{t}: \check{\zeta}_{j} x_{j}-t=0 \quad \text { or } \quad \xi_{n} x_{n}-t=0
$$

upon rotation, with the complex slowness surface $A$. (Thus, the poles are found upon drawing a line along the $\alpha_{n-1}$ - or $\xi_{n-1}$-axis in the hyperplane $X_{t}$ and intersecting it with $A$ ). We will now briefly discuss a simple way of introducing a parametrization of the latter surface, given the one for the real slowness surface.

We consider the decomposition $\operatorname{Re}\{A\}=A_{+}^{\mathrm{gr}} \cup A_{-}^{\mathrm{gr}}$. The holomorphic extension of $f$, and hence $\mathrm{d} \sigma(q, \psi)$, is carried out according to the condition $\operatorname{Im}\{f\}>0$. Let $I_{\psi}$ denote the collection of intervals on the positive real axis in the complex $q$-plane, the endpoints of which are branch points of $f$ at the angle (azimuth) $\psi$ (where $\hat{o}_{\xi_{n}} H=0$ ). Note that there may be branch points in the $q$-plane off the real axis. (They follow directly from the $f^{2}$ vs. $q^{2}$ relations.) Upon integrating over the full slowness hypersurface, however, it follows that contributions from the associated branchcuts cancel. Then $\mathscr{C}=\bigcup_{\psi} I \psi$. Consider the $n$-plet $f(q, \psi)$ with $\operatorname{Re}\{f(0, \psi)\}>0$. Set $\bar{I}_{\psi}=\mathbb{R}_{\geqq 0}-I_{\psi}$ and $\overline{\mathscr{C}}=\bigcup_{\psi} \bar{I}_{\psi}$. There exists a holomorphic extension of $f(q, \psi)$ with $\operatorname{Im}\{f\} \leqq 0$ on $\bar{I}_{\psi}+i 0$ in the complex $q$-plane. This defines $\bar{A}_{+}=\bigcup_{\psi} f\left(\bar{I}_{\psi}, \psi\right) \subset A$ (corresponding with evanescent constituents). In a similar way $\bar{A}_{-}$is defined. We will denote $A_{+} \cup \bar{A}_{+}$by $A_{+}^{*}$, and similarly introduce $A_{-}^{*}$.

Thus, we introduce the $n$-plet of cycles $\partial \gamma=\hat{\partial} \gamma_{+} \cup \hat{\partial} \gamma_{-}$on $A$ through

$$
\partial_{\gamma_{ \pm}}(x, t)=A_{ \pm}^{*} \cap X_{t}
$$

In polar coordinates the cycle $\hat{o} \gamma_{+}$can be parametrized as

$$
\begin{gathered}
\hat{\partial} \gamma_{+}\left(r, \phi, x_{n}, \tau\right)=\left\{q_{+}\left(r, \eta(\phi, \psi), x_{n}, \tau\right), \psi, f\left(q_{+}\left(r, \eta(\phi, \psi), x_{n}, \tau\right), \psi\right) \mid\right. \\
\psi \in([0,2 \pi),[0, \pi), \ldots,[0, \pi))\} .
\end{gathered}
$$

Then $q_{+}\left(r, \eta(\phi, \psi), x_{n}, \tau\right)$ is the $n$-plet of Cagniard-De Hoop contours. The latter contours, with parameter $\tau$, are obtained from the tube of cycles through intersection with the $\left(q, \xi_{n}\right)$-plane (fixed $\left.\psi\right)$ followed by an orthogonal projection on the complex $q$-plane.

In the present representation the time behaviour ("tail") of $E$ is hidden as a (time) parameter in the cycles at a given direction of propagation.

Some Remarks on Causality. The surface $A$ is invariant under complex conjugation in $\zeta$-space (enabling the use of Schwartz' reflection principle in the holomorphic extension of the slowness integral). Under the transformation $\xi \rightarrow \bar{\xi}, \bar{A}_{+}$maps onto $\bar{A}_{-}$(but $A_{+}$maps onto itself) while under the transformation $\xi \rightarrow-\bar{\xi}, A_{+}$maps 
onto $A_{-}$(but $\bar{A}_{+}$maps onto itself). This symmetry implies the equality (restricting the analysis to the spatial half space $x_{n}>0$ with coordinates $x_{j}^{+}$)

$$
\int_{\bar{A}_{+}}\left[\Delta\left(t^{\prime}-\xi_{j} x_{j}^{+}\right) \mathrm{d} \sigma(\zeta)-\Delta\left(t^{\prime}+\overline{\xi_{j} x_{j}^{+}}\right) \overline{\mathrm{d} \sigma(\zeta)}\right]=0,
$$

which is the essential property used in the so-called causality "trick" (Hubral and Tygel [22]); since in $E_{\mathscr{G}}$ we could have replaced the integrand by

$$
\Delta\left(t^{\prime}-\xi_{j} x_{j}-i 0\right) \mathrm{d} \sigma(\xi)-\Delta\left(t^{\prime}+\overline{\xi_{j} x_{j}}-i 0\right) \overline{\mathrm{d} \sigma(\xi)},
$$

we thus could have written

$$
E_{\mathscr{\zeta}}\left(x^{+}, t^{\prime}\right)=\int_{A_{+}^{*}}\left[\Delta\left(t^{\prime}-\xi_{j} x_{j}^{+}\right) \mathrm{d} \sigma(\xi)-\Delta\left(t^{\prime}+\overline{\xi_{j} x_{j}^{+}} \overline{\mathrm{d} \sigma(\xi)}\right] .\right.
$$

The first term on the right-hand side generates the causal component $E^{c}$ of the fundamental solution (this expression follows more directly from the SommerfeldWeyl representation of the fundamental solution) whereas the second term generates the anti-causal component $E^{a}$. Applying the procedure of the previous subsection leads, again, to the introduction of the modified Cagniard contours.

Summary. To obtain a convenient form, we finally permute some coordinates. First, it is understood that in the representations above

$$
\xi_{n}=\xi_{n}\left(\xi_{1}, \ldots, \xi_{n-1}\right) \equiv \tau=\frac{t}{x_{n}}
$$

(parametrization of the slowness surface) while

$$
\xi_{n-1}=\xi_{n-1}\left(\xi_{1}, \ldots, \xi_{n-2}, x_{n}, \tau\right)
$$

(parametrization of the cycle). The rotation and scaling of coordinates yielded

$$
x_{n} \equiv 1, x_{n-1}=\cdots=x_{1}=0 .
$$

A tube $\gamma$ is built up from $(n-2)$-cycles in the time interval $[0, t)$ or in the vertical slowness interval $\left[0, t / x_{n}\right)$; it is a connected piece of the slowness surface. The final representation for $E$ becomes

$$
E=\int_{\gamma} \frac{d \xi_{1} \cdots d \xi_{n-2} d \xi_{n}}{\left(\hat{\partial}_{\xi_{n-1}} H\right)\left(\xi_{1}, \ldots, \xi_{n}\right)}
$$

Permuting the coordinates $\xi_{n-1}$ and $\xi_{n}$ now yields

$$
E=\int_{\gamma} \frac{d \xi_{1} \cdots d \xi_{n-1}}{\left(\partial_{\xi_{n}} H\right)\left(\xi_{1}, \ldots, \xi_{n}\right)}
$$

where the integrand is known as the Leray form.

At this point, it is observed that the construction of the fundamental solution reduces to the construction of cycles on $A$, followed by an integration over these cycles. For isotropic media it is simple to find these cycles; for anisotropic media, however, the construction is far from trivial. In the further analysis, a basis for these 
cycles in the homology group of the associated hypersurface will be found and a differential equation for the integral representation over any basis element will be derived.

\section{The Fundamental Solution Expressed as an Integral over Vanishing Cycles.}

In this section we will show how the geometry of the slowness surface leads to a natural decomposition of the fundamental solution $E$ into integrals over so-called vanishing cycles. These cycles encode the topological information of the wave operator: they depend only on the topology of the singularities (certain critical points) on the slowness surface. To achieve this decomposition, we use the geometrical objects introduced in the two preceding sections. The decomposition of the fundamental solution into integrals over vanishing cycles, has in fact been foreseen already by Petrovsky [38], and also used by Atiyah, Bott and Gårding [8] and by Vasil'ev [43]. This decomposition is non-unique. In principle, one may choose any basis of cycles in the (finite dimensional) space of all vanishing cycles for a given type of singularity, however, as we will show, only for a diagonal integral basis the coefficients of the linear decomposition are integers and can be relatively straightforwardly computed.

Decomposition into Vanishing Cycles. Let us begin with putting the result of the previous section (see also $[8,27,38]$ ) in a more general form:

$$
E=\int_{\gamma} \frac{P\left(\xi_{1}, \ldots, \xi_{n}\right)}{\hat{o}_{\xi_{n}} H\left(\xi_{1}, \ldots, \xi_{n}\right)} d \xi_{1} \cdots d \xi_{n-1},
$$

where $P$ is a degree $D_{P} \leqq D-n-1$ polynomial in $\xi$, where $D$ is the degree of $H$. The integral is over a complex $(n-1)$-dimensional cycle (tube) denoted by $\gamma$ in the $(n-1)$-dimensional algebraic hypersurface $A: H\left(\xi_{1}, \ldots, \xi_{n}\right)=0$, i.e., the cycle is a certain (connected) piece of the slowness surface, oriented such that the coordinate $\xi_{n}$ corresponds to the vertical direction. As was shown in the previous section, the contour $\hat{\partial} \gamma$ arises through intersection $A \cap X_{t}$ and the tube $\gamma$ corresponds with the interval $[0, t)$. The integrand is in fact a closed rational form of degree $(n-1)$ with poles along $\hat{\partial}_{\xi_{n}} H=0$, hence the integral depends on the homology class of $\gamma$ only. This fact will play a central rôle in this section. The quantity $\hat{o}_{\xi_{n}} H$ corresponds to the group velocity in the $n^{\text {th }}$ direction, i.e., the vertical group velocity. Since the cycle depends continuously on the coefficients of $H$, we may use these as deformation parameters of the cycle, without changing the value of the integral.

Define coordinates $\xi_{j}$ on $X_{t}$ such that its local equation is simply $\xi_{n} x_{n}=t$, where $t$ is time. We consider the solutions of the following set of equations

$$
H=0, \hat{o}_{\xi_{1}, \ldots, \xi_{n-1}} H=0,
$$

assuming that the $x_{n}$-axis is oriented along the direction of propagation (observation). In general the roots of these equations will all be different, in which case there are $\mu=D(D-1)^{n-1}$ of them. Label them according to $\xi_{n}^{(i)}, i=1, \ldots, \mu$. These points define singular points (for vertical propagation) on the slowness surface; we will study the topology of the slowness surface around those points in detail.

The level surfaces $H\left(\xi_{1}, \ldots, \xi_{n-1}, \tau\right)=0$ for generic values for $\tau$, considered as a parameter and equal to $t / x_{n}$ (effectively, $\left.x_{n} \equiv 1\right)$, contain $(n-2)$-cycles $\Delta_{i}(\tau)$ 
which have the defining property that they "vanish" when taken along a path $u_{i}$ in the complex $\tau$-plane connecting a generic point $\xi_{n}^{(0)}$ with the point $\xi_{n}^{(i)}$ : upon approaching the point $\xi_{n}^{(i)}$, the cycle $\Delta_{i}$ shrinks to this point. The system of paths $u_{i}, i=1, \ldots, \mu$ is non-intersecting. The cycles $\Delta_{i}, i=1, \ldots, \mu$ are referred to as vanishing cycles. We will explicitly construct these cycles below. The vanishing cycles define $(n-1)$-cycles $\delta_{i}$ on $H=0$ by taking the union of all cycles $\Delta_{i}(\tau)$ as we move along $u_{i}$ from $\xi_{n}^{(0)}$ to $\xi_{n}^{(i)}$. It is a standard fact on integrals of the type (4.1) that the cycles thus constructed are homologous to the cycle $\gamma$ in the sense that there exists the following decomposition:

$$
\gamma=\sum_{i=1}^{\mu} c_{i} \delta_{i}+C_{n-1}\left(\xi_{n}^{(0)}\right)
$$

where the $(n-1)$-cycle $C_{n-1}$ is homologous to zero on $H\left(\xi_{1}, \ldots, \xi_{n-1}, \xi_{n}^{(0)}\right)=0$. This then results in the following decomposition of $E$ over vanishing cycles:

$$
\begin{aligned}
E & =\int_{\gamma} \frac{P\left(\xi_{1}, \ldots, \xi_{n}\right)}{\partial \xi_{n} H\left(\xi_{1}, \ldots, \xi_{n}\right)} d \xi_{1} \cdots d \xi_{n-1} \\
& =\sum_{i=1}^{\mu} c_{i} \int_{\delta_{i}} \frac{P\left(\xi_{1}, \ldots, \xi_{n}\right)}{\partial_{\xi_{n}} H\left(\xi_{1}, \ldots, \xi_{n}\right)} d \xi_{1} \cdots d \xi_{n-1} \\
& =\sum_{i=1}^{\mu} c_{i} \int d \tau \int_{u_{i}} \frac{P\left(\xi_{1}, \ldots, \xi_{n-1}, \tau\right)}{\hat{\partial}_{\xi_{n}} H\left(\xi_{1}, \ldots, \xi_{n-1}, \tau\right)} d \xi_{1} \cdots d \xi_{n-2},
\end{aligned}
$$

where $\xi_{n-1}$ is actually a parametrization for the $(n-2)$-cycle $\Delta_{i}(\tau)$. This is just a rewriting of the formulae at the end of the previous section. Naturally, the $\Delta_{i}(\tau)$ will also depend on $x$; this dependence will be exploited in the later analysis. The numbers $c_{i}$, describing the decomposition of the original integral, are referred to as intersection numbers and are of a topological nature. Generically, the coefficients $c_{i}$ are rational numbers, and are difficult to compute. It is possible, however, to make a special choice of the parameter $\tau$ for which the coefficients become integers. This choice corresponds to an integral basis of vanishing cycles, as we will show. The associated formula for the $c_{i}$ has been derived by Vasiliev [43]. However, the connections with an integral basis and with the invariants of the Picard-Fuchs system are new. In the rest of this section, we will apply elements of singularity theory to make the above decomposition explicit, i.e., we will show how to compute the intersection numbers $c_{i}$.

In order to do so, we will make precise the duality between the slowness surface and the wave front. This will lead naturally to the study of singularities on specific families of hypersurfaces, which are deformations of the original slowness surface. The singularities on the slowness surface are critical points of second (non-degenerate) or higher (degenerate) order. Such points correspond to certain singularities on the wave front. We will show, following $[4,5,7]$, that those singularities can be conveniently studied in terms of critical points of the Legendre transformation relating the slowness surface and the wave front set (the diffraction surface). This will also lead to a complete classification of wave front singularities that can arise in anisotropic elastic media.

The Legendre Transformation and its Singularities. The duality between the projective wave front $\Sigma_{d}$ and $\operatorname{Re}\{A\}$ is described by a Legendre transformation and 
has extensively been discussed in [7]. Since this fact plays a central rôle in our discussion, we will review the argument. Consider $F(\xi, x)$ as a function on the horizontal phase space, formed by the slowness coordinates $\zeta$ and space coordinates $x$, such that

$$
\xi_{j}=\hat{o}_{x_{1}} F \text {. }
$$

(Here, $F$ denotes the travel-time function which replaces the function $S$ of the previous section.) The volume form on the phase space (summation over $j$ )

$$
-d x d \xi=d(-\xi) d x)
$$

closed and nondegenerate. So we may introduce $F$ as the form

$$
d F=d(\zeta d x),
$$

which can be written in our parametrization according to

$$
d F=\sum_{j=1}^{n-1} \xi_{j} d x_{j}+f d x_{n} .
$$

If the medium is homogeneous, integration amounts to (cf. (4.5))

$$
F(\xi, x)=\sum_{j=1}^{n-1} \xi_{j} x_{j}+f x_{n} .
$$

Next we define a momentary (so-called "big") front $\widetilde{\Sigma_{d}}$ in terms of the generating function $F(\xi, x)$ with $x=\left(x_{1}, \ldots, x_{n-1}\right)$ being coordinates in space and $\xi=\left(\xi_{1}, \ldots, \xi_{n-1}\right)$ being coordinates in the dual (momentum) space as the hypersurface in $(2 n-1)$-dimensional space

$$
\widetilde{\Sigma_{d}} \equiv\left\{\left.(\eta, x, \tau) \in \mathbb{R}^{2 n-1}\right|_{\xi}: 0=\frac{\partial F}{\partial \breve{\xi}_{j}}, \quad \eta_{j}=\frac{\partial F}{\partial x_{j}}, \quad \tau=F(\xi, x)\right\} .
$$

The projection $(\eta, x, \tau) \rightarrow(x, \tau)$ is the Legendre transformation and defines the front in the physical $n$-dimensional horizontal space-time. This projection is obtained by solving $\xi$ as a function of $x$ from (4.5). This is a Legendre transformation of the function $f(\xi)$. The surface thus defined is denoted by $\Sigma_{d}$ and corresponds to the momentary front in the physical space.

It is not difficult to see that this construction geometrically corresponds to "taking the polar reciprocal" of the real slowness surface. Namely, consider the graph of the smooth function $f(\xi)$, i.e., let $y=f(\xi), \xi \in \mathbb{R}$, with $f^{\prime \prime} \xi>0$. The Legendre transformation of $f$ is a new function $g$ of a new variable $x$ constructed in the following way. Let $x$ be a given number and consider the line $y=x \xi$. Take the point $\xi(x)$ on the $\xi$-axis such that the vertical distance between the line and $f$ is extremal. That is, for each $x$ the function $F(\xi, x) \equiv \xi x+f(\xi)$ has a maximum at $\xi=\xi(x)$, following from $\partial_{\xi} F=0$, i.e., from $f^{\prime}(\xi)=x$. The function $g$ is now defined as $g(x) \equiv F(\xi(x), x)$. Note that since the second derivative of $f$ does not change sign, the point $\xi(x)$ is unique. The extension to higher dimensions is straightforward.

Let us next apply this to the real slowness surface defined by the equation $H\left(\xi_{1}, \ldots, \xi_{n}\right)=0$ and parametrized by $\xi_{n}=f\left(\xi_{1}, \ldots, \xi_{n-1}\right)$. The function $F$ is, according to the above, defined as 


$$
F(\xi, x)=\sum_{j=1}^{n-1} \xi_{j} x_{j}+x_{n} f\left(\xi_{1}, \ldots, \xi_{n-1}\right) .
$$

Indeed, $F$ is the traveltime along the ray connecting the origin with $x$. Upon dividing by $x_{n} \neq 0$, we may reset

$$
F(\xi, x)=\sum_{j=1}^{n-1} \frac{\xi_{j} x_{j}}{x_{n}}+f\left(\xi_{1}, \ldots, \xi_{n-1}\right) .
$$

Henceforth, we will work exclusively with projective coordinates $x_{j} / x_{n}, j=1, \ldots$, $n-1, x_{n} \neq 0$, denoted by $x_{j}$ as well (we have set $x_{n}=1$ ). Thus, the front in $n$ dimensional space-time is the $(n-2)$-dimensional projective hypersurface

$$
\Sigma_{d}=\left\{(x, \tau) \in \mathbb{R}^{n} \mid \exists_{\zeta(x)}: \hat{\partial}_{\xi_{1}, \ldots, \xi_{n-1}} F=0, \tau=F\right\} .
$$

This is the Legendre dual of the slowness surface parametrized by $f$.

Before, we put this construction of $\Sigma_{d}$ in the context of Petrovsky cycles, introduced in the previous section. The Cagniard-De Hoop contour corresponds to a projection of the tube $\gamma$ onto the complex horizontal slowness plane. The wave arrives exactly where the contour leaves the real axis. The contour is the solution of $F=\tau \in \mathbb{R}$. The complex solutions occur in complex conjugated pairs, hence, the point of intersection with the real axis must be a double root of the equation defining the contour. Thus, at such a point we must have $\partial_{\xi_{1}, \ldots, \xi_{n-1}} F=0$.

It is now evident what the origin of singularities on the wave front is: they are precisely the Legendre transformation of the critical points of $f$ satisfying $\hat{o}_{\xi_{1}, \ldots, \xi_{n-1}} F=0$ in the "big" $(2 n-1)$-space defined above. At these points the function $f$ and its dual $g$ fail to be convex, i.e., at these points at least one of the principal Gaussian curvatures on the slowness surface vanishes and $\xi(x)$ becomes multi-valued. As an example, take the cubic function $f(\xi)=\xi^{3}$. Then $F(\xi, x)=$ $\xi x+\xi^{3}, \xi(x)= \pm \sqrt{-x / 3}$, and hence $\tau=g(x)=(1-\sqrt{3} / 9)(-x)^{3 / 2}$, which has a cusp at the origin in the $(x, \tau)$-plane. The function $F$ is called the generating function of the Legendre transformation. It can be considered as defining a family of "slowness" surfaces described locally by $f$ and parametrized by $\left(x_{1}, \ldots, x_{n-1}\right)$. This is a useful interpretation in the context of singularities. In fact, the function $F$ introduces a deformation of the isolated singularities of the function $f$, such that $F(\xi, 0)=f(\xi)$ parametrizes the original singularity defined by a critical point of $f$.

So far, we discussed momentary fronts, i.e., we did not consider the evolution of fronts in space-time. We will discuss this later in this section.

Singularity Theory. Smooth functions, like $F$, that define isolated singularities have been thoroughly studied [2-7]. For our application, the most important class of functions is the one formed by functions that define isolated, stable singularities, i.e., the ones that are invariant under a local reparametrization of the surfaces. In [7] a complete classification is given of all types of singularities that can occur in Legendre transformations in low dimensions. We will apply this to the Legendre transformations of slowness surfaces in three spatial dimensions. It follows the singularities of fronts in 3-dimensional space fall into three classes. In fact, the critical points on the slowness surface define either a local extremum, an inflection point (i.e., vanishing Gaussian curvature) or a point at which at most the first-order derivative of the curvature along a particular direction vanishes. 
In general, the multiplicity of the isolated critical point of $f(\xi)$ gets resolved into $\mu$ different critical points of the family $F(\xi, x)$ for a generic choice of parameters $x$. However, one usually does not need all the parameters $x$ to lift the degeneracy of a critical point. That is, it suffices to consider a set of $\mu \leqq n-1$ parameters $\lambda$, called versal parameters, for which $F$ has only non-degenerate critical points. In addition, one can show that there always exists local (curvi-linear) coordinates $s(\xi)$ parametrizing the slowness surface close to the critical point such that the function $F$ at such points satisfies the equalities $F=0, \hat{o}_{s} F=0$. Then $F$ is the generating function for the front $\widetilde{\Sigma_{d}}$ introduced earlier. In terms of these coordinates $F$ can be brought into the following standard form:

$$
F(s, \hat{i})=f(s)+\sum_{i=1}^{\mu} \hat{\lambda}_{i} g_{i}(s) .
$$

The functions $g_{i}(s)$ are polynomials in all variables $s_{1}, \ldots, s_{n-1}$ forming a basis in the $\mu$-dimensional vector space of polynomials in $s$ modulo those polynomials generated by the first-order partial derivatives of $f(s)$. The fact that this is a finite $\mu$-dimensional vector space is a standard result. The vector space spanned by $g_{i}(s)$ is sometimes referred to as the local ring of $f$. In general, the evaluation of the canonical transformation involves numerical computations (see also [21] for the evaluation of the canonical form of the phase function in Maslov's theory). We postpone those computations to a future paper. The coordinates $s$ still have the physical interpretation of horizontal slownesses, while the parameters $\lambda$ are horizontal space-time coordinates. In particular, $\hat{\lambda}_{\mu}$ is associated with the polynomial independent of $s$ and represents time. Note that in projective space coordinates one of the $i$ coordinates is set to 1 .

The important point that we want to emphasize here is that for generic choices of $\lambda$ the critical points of $F$ are all distinct (the set of $\lambda$ 's is dense in the set of the original parameters $x$ ). In other words, in a suitably small neighborhood of the critical point of $f$ every analytic function near $f$ can be obtained by analytic changes of variables in $F(s, \lambda)$ for some value of $\lambda$. The form (4.13) of $F$ is called a versal deformation of $f$. The coordinates $s$ are called normal coordinates. We will denote these coordinates by $\xi$ again.

The front generated by $F$ will be in the space of versal parameters $\lambda$. However, the dimension of this space may be lower than the dimension of horizontal space-time. To get a description of the physical front of the "correct" dimension we invoke the Morse lemma which states the following. As $F$ only has non-degenerate critical points, the equation $\hat{o}_{\xi} F=0$ may be solved for $\xi$ as a function of $\lambda$. (This follows from the implicit function theorem.) The Morse lemma states that upon substitution of $\xi(\lambda)$ back into $F$ the latter function can be parametrized in a neighborhood of the critical point in terms of its critical value plus additional parameters according to

$$
F(\xi, \lambda)=F(\xi(\lambda), \lambda)+\sum_{j=1}^{m} z_{j}^{2}-\sum_{j=m+1}^{n-1} z_{j}^{2},
$$

where $m$ denotes the number of positive eigenvalues of the Hessian matrix of $F$ (the determinant of which is non-vanishing) and $z$ has the interpretation of horizontal slowness. From this, it follows that the physical space can be coordinated by $\left(\lambda_{1}, \ldots, \lambda_{\mu} ; \lambda_{\mu+1}, \ldots, \lambda_{n-1}\right)$, such that the additional terms in $F$ are of the form $\lambda_{j} \xi^{2}, j=\mu+1, \ldots, n-1$. 
Table 4.1. Singularities on wave fronts in 3dimensional space

\begin{tabular}{lll}
\hline Type & Normal form & $\mu$ \\
\hline$A_{1}$ & $F=\xi^{2}+\lambda_{1}$ & 1 \\
$A_{2}$ & $F=\xi^{3}+\lambda_{1} \xi+\lambda_{2}$ & 2 \\
$A_{3}$ & $F=\xi^{4}+\lambda_{1} \xi^{2}+\lambda_{2} \xi+\lambda_{3}$ & 3 \\
\hline
\end{tabular}

Now, we investigate what families of functions $F$ may be realized in case of a $x$-independent wave operator in a generically anisotropic medium. To this end, we need the global properties of the slowness surface as discussed in the previous section. Possible points of inflection on the real slowness surface can be detected by considering the condition that a real-valued bitangent exists. A bitangent line is a line tangent to a slowness sheet at two points. Obviously, this can only happen when the slowness sheet is locally not convex. For every bitangent line there will be automatically two inflection points. Note that the bitangent must be a real line as follows from the hyperbolicity condition. A limiting case thus occurs when the two inflection points coincide, as then the bitangent will become complex. This situation occurs at a point $\left(\xi_{1}^{0}, \xi_{2}^{0}\right)$ for which $F$ up to the third-order derivative with respect to $\zeta$ vanishes, implying that $F$ is proportional to $\left(\xi-\xi^{0}\right)^{4}$. As a consequence, the function $F$ is always of a form as listed in Table 4.1. The labelling of the three cases that occur in 3-dimensional space is in accordance with the type of (Weyl) reflection group $^{2}$ that acts on the roots that factorize the polynomial $F$ in $\zeta$. They encode the topological structure of the singularity as we shall now discuss. The wave front is a point of transversal self intersection in the first case $\left(A_{1}\right)$; in the second case $\left(A_{2}\right)$ a cuspidal edge in the $\left(\hat{\lambda}_{1}, \hat{\lambda}_{2}\right)$-plane, dividing the plane into regions of either three real roots of $F=0$ or a real one and two complex conjugated ones. This singularity corresponds to a vanishing Gaussian curvature on the slowness surface. In the third case $\left(A_{3}\right)$ the wave front is known as a swallowtail in $\left(\lambda_{1}, \lambda_{2}, \lambda_{3}\right)$ space. Its intersection with the plane $\lambda_{3}=$ const. is given in Fig. 4.1 along with the location of the roots in the complex $s$-plane of the equation $F=0$ in Fig. 4.2. On the slowness surface this corresponds to a point where the derivative of a Gaussian curvature vanishes.

The equation $F(\xi, \lambda)=0$ defines for generic values of $\lambda$ the non-singular level surfaces of $f$. Introduce the set $\mathscr{S}$ of all $\lambda_{i}, i=1, \ldots, \mu$, and consider for each $\lambda$ the level surface

$$
V_{\lambda} \equiv\left\{\xi \in \mathbb{R}^{n-1}|F(\xi, \lambda)=0,| \xi \mid \leqq \rho\right\},
$$

i.e., we intersect $F=0$ with a small $(n-1)$-ball $B$ of radius $\rho>0$ centered at the critical point of $f$. (This is the region of validity of the normal form representing F.) The surfaces $V_{i}$ are for generic values of $\lambda_{n}$ non-singular and for those values they are all diffeomorphic to the non-singular level surfaces of $f$. The set of $\lambda$ 's for which $V_{\lambda}$ is singular is of codimension 1 in $\mathscr{S}$. This set has an obvious geometrical interpretation: it defines a singularity on a wave front, now parametrized by $\lambda$ instead of $x$. For example, for $A_{2}$, we find that the singular set in $\mathscr{S}$ (corresponding to the cusp) is constrained by the equation

\footnotetext{
${ }^{2}$ As an aside we mention that the manifolds corresponding to the non-regular orbits of the
} Weyl groups are isomorphic to the fronts. 


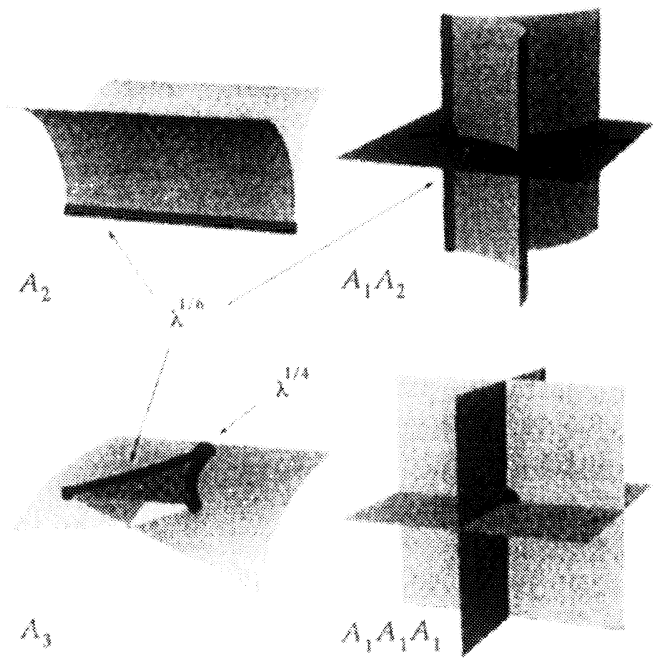

Fig. 4.1. Some possible momentary wave front singularities in 3-D space (see also [3])

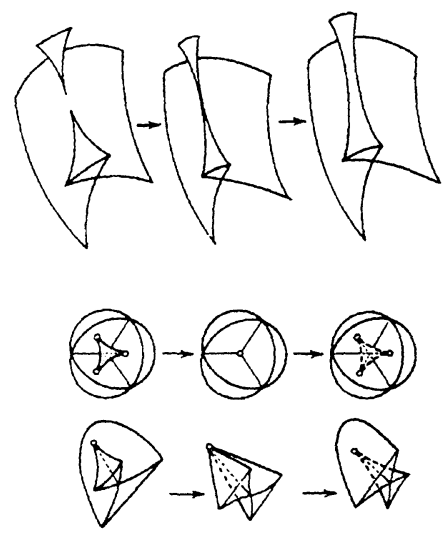

Fig. 4.2. Time evolution of wave fronts in 4-D space time

$$
i_{1}^{3}+c i_{2}^{2}=0, c=\frac{27}{28-6 \sqrt{3}} .
$$

The complement of the front in physical space consists of several disconnected components, see also Fig. 4.1. The components can be characterized by the different sets of parameters $i$ which depend on the actual geometry of the critical point. In each component, however, there is an interval of values for the parameters $\lambda$ for which the location and multiplicities of the critical points do not change qualitatively. It is a property of the singularities in Table 4.1 that only a discrete set of values for the versal parameters is necessary to resolve all the degenerate critical points. This implies that the number of different components is necessarily finite. The solution of the hyperbolic system may be fundamentally different for each component depending on the nature of the front that separates the different regions. We will discuss this later in this section. 
Time Evolution of Fronts in Three Dimensions. Having discussed the possible topologies of the wave fronts in a 3-dimensional (homogeneous) medium, we now turn to the problem of how the fronts evolve in time or $x_{n}$. A front moving in an inhomogeneous medium may change its shape in time, quite generally. To study the possible time evolution of moving fronts, we consider the union of all momentary fronts classified in Table 4.1. This union defines a hypersurface in 4-dimensional space-time. It follows rather easily that this hypersurface is itself a front of a Legendre transformation through a Legendre variety of one dimension higher than the Legendre variety of the momentary front. One considers $x_{n}$ as an additional parameter; the Legendre transformation in the higher dimensional space projects on space-time parametrized by $\left(x_{1}, \ldots, x_{n}, t\right)$ [5]. One thus defines a front in space-time in a similar way as a momentary front, the only difference being that one now considers a one-parameter family of momentary fronts parameterized by $x_{n}$.

This implies that the generating function $F$ of a front moving in space-time can again be put into the general form (4.13), but now with an extra versal parameter, multiplying the constant term, denoting the time function. The fact that a particular versal parameter has the interpretation of time puts a restriction on $F$. Namely, the time function cannot have a critical point, of course. Furthermore, the time should preserve the cuspidal edges of the momentary wave front. (As we work in normal coordinates which are valid close to the singularity, this condition on the time function should only be applied for the singularities on a momentary wave front, and not on the full front). In [5] an important theorem is proved, which states that for a function $F(\xi, \lambda)$ with $\hat{o}_{\lambda \mu} F \neq 0$ ( $\hat{\lambda}_{\mu}$ being time) there exists a diffeomorphism that puts it into the form $F= \pm \lambda_{\mu}$ preserving the front. Now use the expansion of $F$ in (4.14) and assume that $F$ generates a $(\ell<n)$-dimensional cuspidal edge. Let the time function be of the form

$$
t=\lambda_{\mu}+\text { const. or } t=\lambda_{1} \pm \lambda_{\mu+1}^{2} \pm \cdots \pm \lambda_{f}^{2},
$$

where the second expression for $t$ uses the Morse parameters. Hence, in case the normal form of $F$ has less than $n$ parameters $\lambda$, there are multiple choices for the time function.

The singularities on the momentary fronts sweep out a subvariety (a lower dimensional surface) in the hypersurface swept out by the moving front. These singular surfaces are called caustics. The caustics are thus classified using similar techniques as used in the classification of singularities in the Legendre transformations defining the momentary fronts. In case the dimension of the initial space is three, the possible singularities in addition to $A_{1}, A_{2}$ and $A_{3}$ are given in Table 4.2. The time $t$, regarded as an extraction from the normal space-time coordinates, should be a function without critical points and should preserve the "big" front, i.e., the multi-dimensional cuspidal edges of the front. We have listed them in Table 4.2.

The $A_{4}$-type singularity in 4-dimensional space-time corresponds again to a swallowtail, which we will discuss in detail below. The $D_{4}$-type singularities are qualitatively different from the $A_{k}$-type singularities in that they are 2-dimensional rather than 1-dimensional (which is the case for the $A_{k}$-type singularities). They are usually referred to as the "umbilical" singularities [16]. In Fig. 4.3 we have depicted the typical singularities of the caustics in space-time swept by the singularities of momentary wave fronts in 3-dimensional space ([5]).

The swallowtail singularity $A_{4}$ in 4-dimensional space-time is most useful to illustrate the time evolution, as it is related to a common physical phenomenon. 
Table 4.2. Caustics swept in time by singularities of momentary fronts in 3dimensional space (see [7])

\begin{tabular}{|c|c|c|}
\hline Type & Normal form & Time function \\
\hline$A_{3}$ & $F=\dot{\zeta}^{4}+\hat{i}_{1} \dot{\zeta}^{2}+i_{2} \dot{\zeta}+i_{3}$ & $t=\lambda_{3}, t=\lambda_{1} \pm \lambda_{4}^{2}$ \\
\hline $\begin{array}{l}A_{4} \\
D_{4}^{ \pm}\end{array}$ & $\begin{array}{l}F=\dot{\zeta}^{5}+i_{1} \xi^{3}+i_{2} \dot{\zeta}^{2}+i_{3} \zeta+i_{4} \\
F=\eta^{2} \zeta \pm \dot{\zeta}^{3}+i_{3} \xi^{2}+i_{2} \zeta+i_{1} \eta+i_{4}\end{array}$ & $\begin{array}{l}t=i_{4} \\
t=i_{4}, t= \pm i_{3}+i_{1}+i_{2}\end{array}$ \\
\hline
\end{tabular}

Consider a momentary wave front in 3-dimensional space and assume that it has cusp singularities. The cuspidal edges sweep out the caustic. Assume furthermore, that the caustic has a 3-dimensional swallowtail singularity. The caustic is locally diffeomorphic to the polynomial of type $A_{4}$ having a multiple root. The cuspidal edges sweep out a 2-dimensional surface on this hypersurface correponding to a variety diffeomorphic to the $A_{4}$ polynomial having roots of multiplicity of at least 3 . This variety is called an open swallowtail in 4-dimensional space-time. An important result by Arnold [3] states that the time evolution of any cuspidal edge can always upon a canonical transformation be put into the normal form of an open swallowtail, i.e., into a variety given by the zero locus of the $A_{4}$ polynomial with at least two coinciding roots. This greatly facilitates the way the projection onto the physical 3-dimensional space can be carried out: it corresponds to differentiation with respect to $\zeta$. Note that differentiation lowers by one both the degree and the multiplicity of the roots. Thus the problem separates into two parts: first one studies the open swallowtail on the full hypersurface defined by the moving front, and secondly one projects onto the space of degree four polynomials having at least roots of multiplicity two by differentiation.

As time proceeds, the edges disappear into a singularity of type $A_{4}$ in space-time, that, when projected onto 3 -dimensional space, corresponds to an $A_{3}$-type singularity. This type of evolution is easily identified in a physical situation. Namely, suppose a cusp is generated (in a hexagonal medium, say) and moves into an isotropic medium. Then the cusp will eventually evolve into a singular point on the spherical wave front after which it disappears entirely. The singular point is necessarily of type $A_{3}$ in 3-dimensional space. It is interesting that the result in [3] implies that in general the cuspidal edge of a moving front passes the line of self intersection of the swallowtail in 3-dimensional space at two different times. It follows that if the time difference between the arrivals of the two edges is $\Delta \tau$, the time towards the vertex of the swallowtail, i.e., the point after which the cusp has disappeared, scales as $\Delta \tau^{2 / 5}$. Similarly, the $A_{3}$-singularity of the front in space-time leads to a focal point in three-dimensional space, corresponding to a singularity of the type $A_{2}$.

The Decomposition of the Cycle ô. We next describe the cycles in (4.4) in terms

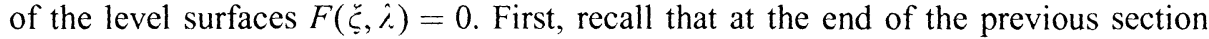
we introduced the cycles as the intersection of $A \cap X_{\tau}$. From (3.42) we draw the conclusion that $A \cap X_{0}$ corresponds with $F(\xi, x)=0$. We have shifted the arrival time of the singularity to the origin. To construct the contours $\partial \gamma$ near the arrivals, we intersect the level surface with a small ball of radius $\rho$ centered at a critical point (which we have taken to be the origin of the affine coordinates $\xi$ ). Thus, we are looking in a neighborhood of a point $x$ located at the front. As we saw already 
this is equivalent to a particular range of values for the versal parameters $\lambda$. The final result for the numbers $c_{i}$ will thus depend on the location at the front or, more precisely, on the component of the complement defined by the front at $x$. Since the front consists of several parts, that is, we can approach the front from various inequivalent directions from the different components of its complement (the space before and after the front) we have to perform the calculation for all components for each singularity separately.

Close to a critical point the function $F$ can be brought into its normal form, i.e., into one of the three representations given in Table 4.1. The contours $\partial \gamma$ are thus contained in the set

$$
A \cap X_{0} \cap B \sim\{F(\xi, x)=0\} \cap B .
$$

Upon constraining the full set of parameters $x$ to the deformation parameters $\lambda$ and having shifted the arrival time to zero, we conclude that the cycles $C_{n-2}\left(\xi_{n}\right) \in B$ are precisely cycles on the surfaces $F(\xi, i)=0$, which we denoted earlier as $V \lambda$. This space is much more convenient, since we can now invoke results by Milnor on properties of the homology group formed by these cycles.

According to Milnor [31] the space of cycles $C_{n-2}$ is of finite dimension $\mu$, and any $(n-2)$-cycle can be expanded into a suitably defined basis of cycles, such that the coefficients of this expansion are all integers. More precisely, the $\mu$ dimensional homology of the fibers $V_{i}$ for generic values of the parameters $i$ form a $\mu$-dimensional lattice:

$$
H_{n-2}\left(A \cap X_{0} \cap B\right)=H_{n-2}\left(V_{\lambda}\right)=\mathbb{Z}^{\mu} .
$$

A basis in this homology is given by a system of $\mu$ cycles $\Delta_{i}$ which vanish along suitable chosen paths connecting a given non-critical value of $F$ with a critical one. Such a basis is referred to as a basis of vanishing cycles. Milnor's theorem thus states that there exists a basis of vanishing cycles such that the cycle $\partial \gamma$ on a level surface of $f$ can be decomposed into vanishing cycles drawn on the level surfaces defined by $F=0$, such that the coefficients are all integers. That is, there exists a basis of cycles such that the intersection numbers $c_{i}$ in (4.4) are all integers. In a generic basis the numbers $c_{i}$ are rational numbers; only for a special choice of basis cycles, the numbers are integers. How to determine this particular basis will be postponed until the next section.

We are now in a position to actually construct such systems of paths and the associated cycles. First we divide the critical values of $F(\xi, i)$ into two sets: the real critical values, and the ones that come in complex conjugated pairs. In order to make contact with the original integral $E$ it is obvious that we have to decide on a particular orientation of those cycles that correspond to the real critical values: they have to be in agreement with the chosen orientation of the slowness sheets. Assume that the origin is a non-critical value of $F$. Let $U$ be that portion of the complex plane that contains all the critical values. Now connect the origin with any real critical point with a path in $U$ such that it always consists of points whose imaginary parts are in absolute values less than the absolute values of the imaginary parts of any other complex critical point. Further, paths joining the origin with critical points that come in complex conjugated pairs, are conjugated and always contained in either the positive half plane or negative half plane according to the imaginary part of the critical value. We furthermore require that they intersect the real axis transversally (in agreement with Schwarz's reflection principle). 
Let the appropriate system of critical paths $u_{i}(\hat{\lambda}), i=1, \ldots, \mu$, in the $\tau$-plane, obeying the above conditions, be parametrized by $i$. Applying (4.14), we find that $F$ is always locally parabolic away from the singularity. The vanishing sphere associated with the critical point $Z_{i}$ of $F$ is a real $(n-1)$-sphere $S_{i}$ with a $\hat{i}_{\text {-dependent }}$ radius

$$
\sum_{i=1}^{n-1} \zeta_{j}^{2}=u_{i}(i)-Z_{i}
$$

Note that this sphere shrinks to a point as $\lambda \rightarrow 0$. The orientation of this sphere and hence of $S_{i}$ is such that its volume form is positive with respect to the volume form orienting the (real) slowness surface. The homology class in $H_{n-2}\left(V_{\lambda}\right)$ represented by $S_{i}(\lambda)$ is the vanishing cycle corresponding to the path $u_{i}$ and is denoted by $\Delta_{i}$. The system $\left\{\Delta_{i}\right\}_{i=1}^{\mu}$ forms a basis in this homology group. It is with respect to this basis that we will compute the numbers $c_{i}$ in (4.4).

There is a lot of freedom in choosing a basis of vanishing cycles, which is reflected in the invariance of this basis under automorphic transformations of the homology group $H_{n-2}$, i.e., the isometries of the Milnor lattice. These automorphisms are referred to as monodromy transformations. Rather than discussing this in detail, we will simply list the results necessary for the computation of the intersection numbers $c_{i}$. It turns out that a monodromy transformation can be described analytically by studying the properties of the vanishing cycles implied by the following transformations. Consider the transformation of the path $u_{i}$ to the composite path that goes along $u_{i}$ starting at a given non-critical value, then anti-clockwise around the critical point $Z_{i}$ and then back along $u_{i}$. Under such transformations the critical points of $F$ are permuted, but a vanishing cycle, i.e., a contour on a nonsingular level surface which vanishes upon taking the parameters to zero, remains invariant. This enables one to write down a canonical representation of the vanishing cycles in terms of a suitably chosen parametrization of the level surface. This is reflected in the so-called Picard-Lefschetz formula, which gives a canonical form of the inner product on the vanishing cycles, denoted by $(.,$.$) . Applying this formula$ yields the following representation of the monodromy operator $h_{i}$ corresponding to a transformation encircling the $i^{\text {th }}$ critical point:

$$
h_{i}(\beta)=\beta+(-1)^{n(n-1) / 2}\left(\beta, \Delta_{i}\right) \Delta_{i},
$$

where $\beta$ is an arbitrary cycle of dimension $n-2$. It is thus seen that the monodromy group acts as a (pseudo) reflection, $h_{i}\left(\Delta_{i}\right)=-\Delta_{i}$, and it is the identity on the hyperplane orthogonal to $\Delta_{i}$. In general, the intersection product is hard to compute explicitly, and only for a few classes of singularities there exist explicit results. However, for simple singularities, amongst which are those of Table 4.1 , it can be computed easily. In fact, for singularities defined by

$$
f(\xi)=\xi^{k+1},
$$

it can be shown that one can bring a basis of vanishing cycles into the following form:

$$
\left(\Delta_{i}, \Delta_{i+1}\right)=-1, \quad\left(\Delta_{i}, \Delta_{j}\right)=0 \text { for }|i-j| \geqq 2 .
$$

Furthermore, we can construct the composition of all operators $h_{i}$, denoted by $h_{*}$, which thus describes a loop based at a non-critical value, encircling all critical values once. It turns out that, in case of $A_{k}$-type singularities, such an operator has eigenvalues given by the roots of unity $\exp (2 \pi i j / k), j=1,2, \ldots, k$. 
For the simple singularities of type $A_{k}$ the resulting reflection groups are just the permutation groups on respectively two or three elements, which are the Weyl groups of the Lie groups $A_{1}, A_{2}$ and $A_{3}$. For us the most important results are that the intersection indices appearing in (4.4) are all integers in the basis (4.19). Furthermore, they depend on the particular component of the complement of the front. In terms of monodromy this is rephrased as follows. The front itself corresponds to the "mirrors" of the monodromy group, i.e., to the so-called non-regular orbits. These mirrors separate the Weyl chambers, which are the space in between the mirrors, i.e., the points on the regular orbits of the monodromy group. The $c_{i}$ thus depend on the particular Weyl chamber chosen.

Let us now finally compute explicitly these indices using a more general result in [43]. As shown there, it is possible in each component of the complement of the wave front, to organize the intervals of the versal parameters such that the degenerate critical values are zero, while the non-degenerate critical values are all real. Hence we only need to compute the indices for the cases $Z_{i}$ being positive or negative.

In fact, using the description of the vanishing cycles (4.18) it follows that for three spatial dimensions the intersection of the contour $\hat{\gamma} \gamma$ with the vanishing cycle $\Delta_{i}$ corresponding to the $i^{\text {th }}$ real critical point is given by

$$
\begin{aligned}
& Z_{i}>0: c_{i} \equiv\left(\Delta_{i}, \hat{\partial} \gamma\right)=-\left(1+(-1)^{3-k}\right)-\sum_{j}\left(\Delta_{i}, \Delta_{j}\right), \\
& Z_{i}<0: c_{i} \equiv\left(\Delta_{i}, \partial \gamma\right)=-\left(1+(-1)^{k}\right)+\sum_{j}\left(\Delta_{i}, \Delta_{j}\right) .
\end{aligned}
$$

For complex conjugated critical values one has

$$
Z_{\bar{i}}=\bar{Z}_{i}: c_{i} \equiv\left(\Delta_{i}, \hat{\partial} \gamma\right)=\left(\Delta_{\bar{i}}, \hat{\partial} \gamma\right)=\left(\Delta_{i}, \Delta_{\bar{\imath}}\right) .
$$

The sum over $j$ is over those vanishing cycles associated with real critical values that lie in between the origin and $Z_{i}$. The original cycle $\partial \gamma$ thus has the following decomposition in terms of vanishing cycles

$$
\partial \gamma=\sum_{i=1}^{\mu} c_{i} \Delta_{i}
$$

with the intersection numbers $c_{i}$ given in (4.24)-(4.25).

For the different disconnected regions shown in Fig. 4.3 the locations, in the complex plane, of the roots of the level set of $f$ are different, hence the $c_{i}$ will be different. It may happen that for a given component all the $c_{i}$ vanish, thus implying that the solution is completely vanishing. Such regions are called local lacunae.

Vasiliev has computed in [43] for the $A, D$ and $E$ singularities up to $n=7$ dimensions how many different regions may occur for a given front. For the singularities occurring for moving fronts in 4-dimensional space-time this number is one. This is the shaded region in Fig. 4.4. Furthermore, a simple calculation reveals that if these regions are approached from the directions orthogonal to the limiting tangents denoted by $L$, the lacuna is penetrated.

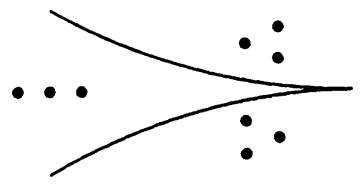

Fig. 4.3. Location of the roots of $F=0$, with $F$ of type $A_{2}$ 


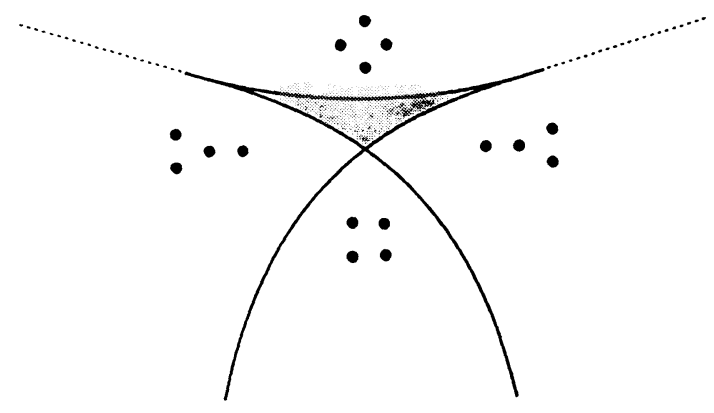

Fig. 4.4. Location of the roots of $F=0$, with $F$ of type $A_{3}$. The shaded region is a lacuna, which is penetrated if it is approached along the dotted lines

\section{A Differential Equation for the Fundamental Solution}

The main point of the previous section was that the geometry of momentary fronts in space as well as of moving fronts in space-time are conveniently studied in terms of the zero level surface of functions of the form (4.13) that generate the front in space or space-time according to whether $F$ is chosen from Table 4.1 or from Table 4.2. The function $F$ has the property that if all versal parameters are vanishing $F$ reduces to a function $f(\xi)$, which we used to parametrize the slowness surface.

In this section we will study the fundamental solution $E$ in space-time expressed as a linear combination of integrals over vanishing cycles associated with the singularity of $f$ whose versal deformation $F$ generates the front in space-time ( $F$ is of the form listed in Table 4.2). Thus $F$ describes the caustic set swept out in space-time by the cuspidal edges of the momentary wave fronts arising from inflection points on the real slowness surface discussed in Sect. 3 and 4. From our discussion so far it is not obvious that it is precisely the caustic set of the moving front that determines the global asymptotic behavior of the fundamental solution in space-time. This can be shown by rewriting the original integral over the slowness surface as an oscillatory integral in the time-frequency domain. In the exponent in the oscillatory integral appears the phase function which, in fact, is (in normal coordinates) the generating function $F$ of a moving front in space-time. Upon applying the well-known method of stationary phase one concludes that the integral is dominated in the high-frequency limit by the (non-degenerate) critical points of $F$. Rather than discussing this here (see, e.g., [16]) we will concentrate on the integrals over vanishing cycles.

As we emphasized in the previous section, the decomposition of the fundamental solution into integrals over vanishing cycles is valid for any choice of the versal parameters. However, only for a special choice of basis vectors in the space of versal parameters (as given for the $A_{k}$ singularities in the previous section) the intersection indices $c_{i}$ are the integers given in (4.24)-(4.25). To use the latter equations, we have to find the appropriate parametrization of versal deformations in terms of the original arbitrarily chosen parameters. The homology basis must be integral [28] and can be characterized by the fact that the integrals over the vanishing cycles on the non-singular level surfaces parametrized by these coordinates satisfy the system of non-linear differential equations associated with the monodromy invariants of the 
Picard-Fuchs equation. These invariants are uniquely determined by the topological type of the singularity.

Our aim in this section is to exploit the invariants of the Picard-Fuchs system to derive global asymptotic solutions of the hyperbolic system exhibiting arbitrary caustic sets. Here, we will derive the asymptotics from the Picard-Fuchs system. Although the Picard-Fuchs differential equation is well known amongst mathematicians $[19,26]$, the relation between the invariants of the associated non-linear system and the monodromy group of the singularity is apparently new, see the third reference in [28]. We will show the Picard-Fuchs system for an integral over a vanishing cycle. The solution of this system determines the fundamental solution near its geometrical arrivals.

We will carry out the computations for the nontrivial singularities of Table 4.2. First, we will explain why the integral over a vanishing cycle satisfies a linear differential equation. Let $f\left(\xi_{1}, \ldots, \xi_{n-1}\right)$ be the vertical slowness having an isolated singularity at the origin of criticality $\mu$. Let $F(\xi, \lambda)$ be a versal deformation, with the property $F(\xi, 0)=f(\xi)$. Consider also the so-called Milnor fibration $\pi: V \rightarrow \mathscr{S}$ whose fibers $V_{\lambda}$ over the space $\mathscr{S}$ of versal deformation parameters are isomorphic to the level surface $F(\zeta, \lambda)=0$. This level surface is diffeomorphic to the non-singular level surface $F(\xi, \hat{i})=\tau$. The parameter $\tau$ depends implicitly on $i$; it reduces to $\tau=\xi_{n}$ if all (projective) versal parameters vanish. Thus, $\tau$ defines a "distance" function along a curve in $\mathscr{S}$ intersecting the singular set $\Sigma_{d}$ at most at isolated points. If the curve in $\mathscr{S}$ intersects $\Sigma_{d}$ transversally then this "distance" corresponds to the time function in the normal coordinates defined in the previous section.

Our integrals are over a rational closed $(n-2)$-form, which define locally constant classes in the homology $H_{n-2}\left(V_{\lambda}\right)$. This allows us to put the cycle in a canonical form without changing the value of the integral. If we stay close enough to the critical point, one may thus differentiate under the integral with respect to the versal parameters. For convenience, we repeat here the form of the integral over a vanishing cycle (cf. (4.1))

$$
I=\int_{\Delta}\left(\frac{P d \xi}{\partial_{\xi_{n}} H}\right)
$$

The integrand has poles precisely at the $\mu$ critical points of $H$ in the $\xi_{n}$-direction. The differential equation which we are about to derive is actually a calculation in the cohomology (the Poincare dual) of $H_{n-2}\left(V_{\lambda}\right)$. It is well known that the cohomology of projective hypersurfaces can be calculated from its rational differential forms having poles at infinity. To do the actual computation a bound on the order of the poles is required. Such bounds can be determined using the work of Griffiths [19]. The order of the pole of the rational integrand in (5.1), which a priori has a pole of arbitrary order, can be put in some canonical form in which it has a pole of order one. This reduction-of-pole property is extensively discussed in [19]. Here, we will only briefly mention the idea, which is as follows. The middle cohomology of the complex hypersurface $V_{\lambda}$ in $\mathbb{C I P}^{n-2}$ is described by rational differentials in $\mathbb{C P}^{n-1}$ having poles of arbitrary order along $V_{\lambda}$. Each form $P d \xi / \partial_{\xi_{n}} H$ defines a cohomology class by considering its residue on $V_{\lambda}$ as follows. The $(n-2)$-cycle $\Delta_{i}(\lambda)$ on $V_{\lambda}$ can be made into an $(n-1)$-cycle $\delta_{i}(\lambda)$ by considering the tube over $\Delta_{i}(\lambda)$, i.e., $\delta_{i}(\lambda)$ is a small cylinder erected along the normal over $\Delta_{i}(\lambda)$ in $\mathbb{C P}^{n-1}$. Then we can define the residue associated with the first-order pole as 


$$
\int_{\Delta_{i}(\lambda)} \operatorname{Res}_{V_{i}}\left(\frac{P d \xi}{\hat{\partial}_{\xi_{n}} H}\right)=\frac{1}{2 \pi i} \int_{\delta_{i}(\lambda)}\left(\frac{P d \xi}{\hat{o}_{\xi_{n}} H}\right) .
$$

We suppress the index $i$ attached to the vanishing cycle from now on.

Recall that the value of the integral on the right-hand side does not change if we add to integrand an exact differential form having poles on the hypersurface $H=0$. This can be used to lower the order of the pole of the integrand (5.1) on $V_{\lambda}$ as follows. Let $l$ be the sum of the weights of the quasi-homogeneous polynomial defined by the principal symbol $H$ (of degree $D$ ). Consider quasi-homogeneous polynomials $A_{j}, j=1, \ldots, n-1$, of degree $D+l_{j}-l$, where $l_{j}$ is the weight of the $\xi_{j}$ in $A_{j}$. Define the $(n-3)$-form

$$
\phi=\frac{1}{\hat{\partial}_{\xi_{n}} H} \sum_{i<j}\left(l_{i} \xi_{i} A_{j}-l_{j} \xi_{j} A_{i}\right) d \check{\zeta}_{1} \cdots \widehat{d \zeta}_{i} \cdots{\widehat{d \xi_{j}}} \cdots d \xi_{n-1} .
$$

Its differential is defined as

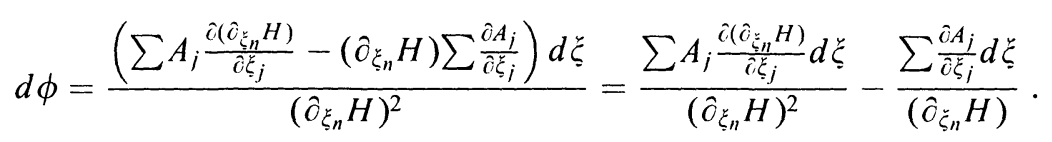

This shows that any form of which the numerator can be written as a linear combination of partial derivatives $\hat{\partial}_{\xi_{j}}\left(\hat{\partial}_{\xi_{n}} H\right)(\xi)$, is equivalent up to (rational) exact forms to a rational form with a smaller order pole. By this reduction technique, one can put the integrand in a canonical form, more precisely, one associates a specific cohomology class to such integrands.

Let us, before we proceed, illustrate this method by way of a simple example, where the integral (5.2) is simply a contour integral in the complex plane. The residue in (5.2) becomes the familiar Cauchy residue. Now assume that the integrand has a pole of order two at $\xi=0$, say. Then it has a Laurent series expansion of the form $\left(b / \xi^{2}+c / \xi+\cdots\right) d \xi$ with constants $b, c$. Let $\phi=b / \xi$. Then

$$
\frac{P d \xi}{\partial \xi_{n} H}+d \phi \simeq\left(\frac{c}{\xi}+\cdots\right) d \xi
$$

has only a first-order pole. Proceeding in this way, we can reduce the pole at $\partial_{\xi_{n}} H=0$ of the integrand to one of order one at any point on the hypersurface for which $H=0$. This brings the rational form in its canonical form.

It is obvious that in general the integral over a vanishing cycle as a function of $\lambda_{i}$ will be multivalued due to the nontrivial monodromy. Thus, the $\mu$-dimensional vector

$$
I\left(\lambda_{1}, \ldots, \lambda_{\mu}\right) \equiv\left(\int_{\delta_{1}} \frac{P d \xi}{\partial_{\xi_{n}} H}, \ldots, \int_{\delta_{\mu}} \frac{P d \xi}{\partial_{\xi_{n}} H}\right)
$$

will be multivalued as well. We will now show that this vector satisfies a linear differential equation in $\lambda_{i}$ with unique holomorphic coefficient functions $p_{i}(\lambda)$, whose solutions are linear combinations of the integrals (5.5). We remark here, that in principle there are different parameters $\lambda_{i}$ depending on the type of singularity. The singularities relevant to us, all have the property that the parameter $\lambda_{i}$ is always such that it resolves the singularity; such singularities are referred to as modality zero singularities. We will refer to $\lambda_{i}$ as being a relevant parameter. In contrast with 
this, there are also singularities for which there exist parameters $\lambda_{i}$ that continuously deform the singularity without changing its type, i.e., without resolving it. In such a case $\hat{i}_{i}$ is referred to as a modulus. Also in this case the integral $I\left(\hat{i}_{i}\right)$ satisfies a linear Picard-Fuchs equation, which can be derived in a similar way as for a relevant parameter. We consider first the case of just one parameter $\lambda$.

The Single-Parameter Case. We will discuss the Picard-Fuchs system for functions $F$ of the type $A_{1}$ (a non-degenerate critical point) and of the type $A_{2}$. In the latter case the momentary wave front arises from a turning point.

Recall that the cohomology class defined by the cycle $\delta$ does not change upon varying $\lambda$ locally, so the differentiation with respect to $\lambda$ can be taken under the integral:

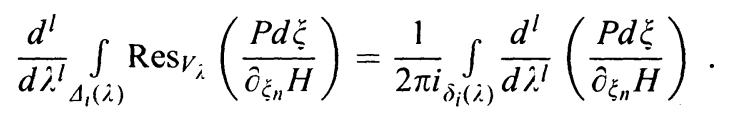

Now construct the vector $I^{j}(i)$ by taking the $j^{\text {th }}$ derivative with respect to $\lambda$, i.e.,

$$
I^{j}(\lambda) \equiv\left(\frac{d^{j}}{d \lambda^{j}} \int_{\delta_{1}} \frac{P d \xi}{\partial_{\xi_{n}} H}, \ldots, \frac{d^{j}}{d i_{i}^{j}} \int_{\delta_{\mu}} \frac{P d \xi}{\partial_{\xi_{n}} H}\right) .
$$

The vector spaces $W_{i}$ formed by all vectors $I^{j}, j \leqq i$, must have constant dimension as function of $\lambda$, as the integrands are all closed $(n-2)$-forms in $\lambda$. Also, the dimension $d_{i}=\operatorname{dim} W_{i}$ of these spaces cannot exceed $\mu$. Thus there will be a smallest number $s \leqq \mu$, such that

$$
I^{s}(\lambda)=-\sum_{j=0}^{s-1} p_{j}(\lambda) I^{j}(\lambda)
$$

That is, the vector $I$ satisfies a differential equation of the type

$$
\left(\frac{d^{s}}{d \hat{i}^{s}}+\sum_{i=0}^{s-1} p_{i}(\lambda) \frac{d^{i}}{d \lambda_{i}^{i}}\right) I=0
$$

Such an equation is referred to as a Picard-Fuchs equation [6]. The unknown coefficient functions are determined by taking successive derivatives with respect to $\lambda$ and making repetitive use of the pole-reduction technique discussed earlier (to carry out the partial integration, effectively). An important property of Picard-Fuchs equations is that they can have at worst regular-singular points, at which the coefficient functions may develop poles due to the monodromy around such poirts. By multiplying the Picard-Fuchs operator

$$
\frac{d^{s}}{d \lambda^{s}}+\sum_{i=0}^{s-1} p_{i}(\lambda) \frac{d^{i}}{d \lambda^{i}}
$$

by $i^{s}$, the equation takes the form

$$
\left(\left(\lambda \frac{d}{d \lambda}\right)^{s}+\sum_{i=0}^{s-1} q_{i}(\lambda)\left(\lambda \frac{d}{d \lambda}\right)^{i}\right) I=0,
$$

the coefficient functions of which are now holomorphic also at the regular-singular points. (Upon changing $\lambda$, the integral plus all its derivatives changes according to 
the monodromy operator, in the same way.) We will see shortly that the order $s$ of the equation is fixed by the monodromy group. (A more extensive analysis, valid for more general types of singularities, is given in [28].)

As a simple example, consider the $A_{1}$ singularity with

$$
F=\xi^{2}+i .
$$

Since $\mu=1$, we only have one point representing the zeroth vanishing homology class. The differential equation in this case must be of first order, viz.

$$
\frac{d I}{d \lambda}-\frac{1}{\lambda} I=0
$$

hence $I=$ const. $\lambda^{-1}$. This gives the asymptotic expansion near the critical point. The full solution $E$ can in this case easily be obtained. From the above we conclude that it must be of the form

$$
i^{-1} g(\hat{i})
$$

with $g(\hat{\lambda})$ a holomorphic function depending on $\lambda$. Since $F$ is quadratic, $I$ is in fact an integral over an $(n-2)$-sphere of radius $\sqrt{\lambda}$, so that

$$
g(\lambda)=\frac{n-1}{2} \frac{\pi^{(n-1) / 2}}{\Gamma((n-1) / 2+1)},
$$

which is half of the surface of the unit $(n-2)$-sphere, independent of $\lambda$. (Compare this with the results in Sect. 3 for the HPL formula.)

The quadratic case $A_{1}$ is generic, i.e., away from the degenerate critical points the slowness surface can always be parameterized by a function $F$ quadratic in $\zeta$. The points corresponding to degenerate critical points, i.e., points where $F$ is of type $A_{2}$ or $A_{3}$, are special. In those cases $g$ does depend on $\lambda$ and likewise the full solution is more complicated, although its asymptotics may be readily obtained as we will see below. For the $A_{2}$-type singularity,

$$
F=\xi^{3}+\lambda \xi,
$$

and the differential equation reads (after bringing it into its familiar form)

$$
\left(\frac{d^{2}}{d \hat{\imath}^{2}}-\frac{1}{3} \lambda\right) I=0 \text {. }
$$

Two independent solutions to this equation are the Airy functions $\operatorname{Ai}(i)$ and $\operatorname{Bi}(\lambda)$. It is easy to verify that $I(\lambda)$ has a (holomorphic) limit $\lambda \rightarrow 0$. In fact, by the techniques outlined above we find for any isolated singularity (not only the simple ones) a Picard-Fuchs equation whose solution has a holomorphic limit $\lambda \rightarrow 0$, and thus gives an exact solution for $I$ at the singularity. The Airy function is a wellknown example studied extensively in geometrical optics. Usually one arrives at this solution using Maslov theory.

Here, we arrived at such solutions using a differential equation. As we will now discuss, this has the advantage that it illuminates the topological aspects of the asymptotics of $I$ near the singularity. Namely, we investigate a solution that manifestly is invariant under multiplication by an analytic function of $i$. As we argued at various places throughout this paper, this expresses the freedom in choosing a basis of vanishing cycles. In order to make this invariant property of the solution at the 
front manifest, we associate to the linear differential equation (5.14) the following well-known non-linear equation. For any two independent solutions $I_{1}(\lambda), I_{2}(\lambda)$, the quotient

$$
\zeta(\lambda) \equiv \frac{I_{1}(\lambda)}{I_{2}(\lambda)}
$$

satisfies the differential equation

$$
\{\zeta, \lambda\}=-\frac{2}{3} \lambda,
$$

where the left-hand side denotes the Schwarzian differential defined as

$$
\{\zeta, \lambda\} \equiv \frac{\zeta^{\prime \prime \prime}}{\zeta^{\prime}}-\frac{3}{2}\left(\frac{\zeta^{\prime \prime}}{\zeta^{\prime}}\right)^{2} .
$$

It can easily be verified that the Schwarzian differential is unchanged if one replaces $(5.14)$ by the linear equation for $g(\lambda) I(\lambda)$ with $g(\lambda)$ an arbitrary smooth function. Furthermore,

$$
\{\zeta, \hat{i}\}=0 \text { if and only if } \zeta=\frac{a \hat{\imath}+b}{c \hat{\imath}+d}
$$

with $a, b, c, d \in \mathbb{C}$ and $a d-b c \neq 0$. As the monodromy group of (5.14) is generated by such linear fractional transformations, we conclude that the solution is invariant under the monodromy as required.

The monodromy invariant solution of the non-linear equation gives the preferred decomposition of the integral $E$, for which the expansion coefficients $c_{i}$ in (4.4) are the integers given by (4.24)-(4.25).

The Multi-Parameter Case. We will now derive a Picard-Fuchs equation for general directions and write it as the so-called zero-curvature condition for a flat connection known as the Gauss-Manin connection. The approach followed here is explicitly discussed in the third reference in [28]; we mention it here briefly for completeness. Let $\mu$ be the multiplicity of the isolated singularity on $H(\xi)=0$ at $\xi=0$, and let $\lambda_{1}, \ldots, \lambda_{\mu-1}$ be the versal parameters multiplying non-constant polynomials in $\xi$ and let $\lambda_{\mu}$ be the unique parameter multiplying the constant term. (Recall that this parameter has the interpretation of time function in normal coordinates.) Introduce

$$
I_{\alpha}^{(l)}=(-1)^{l+1} \Gamma(l+1) \int_{\delta} \frac{P_{\alpha} d \xi}{\left(\partial_{\xi_{n}} H\right)^{l+1}},
$$

where we allowed for a slight generalization to include arbitrary powers $l$ in the denominator. The normalization factors are only convenient for the calculation, but inessential for the argument. The objects $I$ satisfy the following differential equations

$$
\begin{aligned}
& \hat{\partial}_{\lambda_{\mu}}^{k} I_{\alpha}^{(l)}=I_{\alpha}^{(l+k)}, \quad k \in \mathbb{Z}, \\
& \hat{\partial}_{i_{i^{\prime}}} I_{\alpha}^{(l)}=\sum_{k=-1}^{\infty} B_{i^{\prime} \alpha}^{(k) \beta}\left(\lambda^{\prime}\right) I_{\beta}^{l-k}, \\
& \lambda_{\mu} I_{\alpha}^{(l)}=-\sum_{k=0}^{\infty} A_{\alpha}^{(k-2) \beta}\left(\lambda^{\prime}\right) I_{\beta}^{(l-k)},
\end{aligned}
$$


where $i^{\prime}=\left(\hat{i}_{i^{\prime}}\right), i^{\prime}=1, \ldots, \mu-1$. Next, we apply the Laplace transformation in $i_{\mu}$

$$
I\left(., i_{\mu}\right) \rightarrow \int_{-\infty}^{0} I\left(., i_{\mu}\right) \exp \left(i_{\mu} / z\right) d i_{\mu},
$$

so that

$$
\begin{aligned}
& \hat{o}_{\lambda_{\mu}} \rightarrow \frac{1}{z}, \\
& i_{\mu} \rightarrow-z^{2} \frac{d}{d z},
\end{aligned}
$$

and

$$
I_{\chi}^{(l)}(\hat{i}) \rightarrow I_{\chi}^{(l)}\left(z, \hat{i}^{\prime}\right)
$$

As a result the last two equations in (5.20) reduce to

$$
\begin{gathered}
\left(\delta_{\alpha}^{\beta} \hat{\partial}_{i^{\prime}}-\sum_{k=-1}^{\infty} z^{k} B_{i^{\prime} \alpha}^{(k) \beta}\left(\hat{\iota}^{\prime}\right)\right) I_{\beta}^{(l)}\left(z, \hat{\iota}^{\prime}\right)=0, \\
\left(\delta_{\alpha}^{\beta} \hat{o}_{z}-\sum_{k=-2}^{\infty} z^{k} A_{\alpha}^{(k) \beta}\left(i^{\prime}\right)\right) I_{\beta}^{(l)}\left(z, i^{\prime}\right)=0,
\end{gathered}
$$

which reveals that the matrices $B_{i^{\prime}}^{(k)}$ and $A^{(k)}$ are flat connections of a vector bundle over the space $\mathscr{S}$, whose sections are $I_{\alpha}^{(l)}$. The remarkable thing is [28] that one may further reduce this matrix system of first-order equations to the case in which

$$
B_{i^{\prime}}^{(k) \beta}=0, \quad k \geqq 1 .
$$

Define the covariant differentials

$$
\begin{aligned}
& D_{i^{\prime} \alpha}^{\beta}=\delta_{\alpha}^{\beta} \partial_{i_{i^{\prime}}}-B_{i^{\prime} \alpha}^{(0) \beta}\left(\hat{i}^{\prime}\right), \\
& C_{i^{\prime} \alpha}^{\beta}=B_{i^{\prime} \alpha}^{(-1) \beta}\left(\hat{\iota}^{\prime}\right) .
\end{aligned}
$$

Then the compatibility equation for the system (5.25)-(5.26) to have a solution can be cast in a so-called zero curvature condition

$$
\left[D_{i^{\prime}}-z^{-1} C_{i^{\prime}}, D_{i^{\prime}}-z^{-1} C_{i^{\prime}}\right]=0 \text { for all } z,
$$

which can also be written as the two simultaneous equations

$$
\left[D_{i^{\prime}}, D_{j^{\prime}}\right]=\left[C_{i^{\prime}}, C_{j^{\prime}}\right]=0 .
$$

Equation (5.31) is the multi-parameter analogue of the Picard-Fuchs equation (5.9) derived in the single parameter case. It specifies coordinate functions in the deformation parameters $\lambda$ on $\mathscr{S}$ for which the matrices $A$ and $B$ are holomorphically flat. Such matrices define a Gauss-Manin connection. Each corresponding section describes the solution at the front for a different direction, specified by the parameter $i^{\prime}$. It is conceivable that for different intersections with $\Sigma$ one finds different asymptotic behavior; then there will be a "most singular" direction. 
We will now give the equations for the $A_{3}, A_{4}$ and $D_{4}$ singularities and discuss the rôle of the various directions parametrized by the different versal parameters. In case of the $A_{3}$ singularity there are two deformation parameters, viz.

$$
F=\xi^{4}+i_{1} \xi^{2}+\lambda_{2} \xi .
$$

Thus we get a system of partial differential equations for $I(\lambda)$. One finds

$$
\frac{\hat{o}^{2} I}{\partial \hat{i}_{1}^{2}}+i \frac{\partial I}{\partial \hat{i}_{2}}=0
$$

The other equation is found from $\hat{o}_{\xi} F=0$ :

$$
\left(4 \frac{\partial^{3}}{\partial \hat{i}_{2}^{3}}-2 \hat{\lambda}_{1} \frac{\hat{\partial}}{\partial \lambda_{2}}+i \hat{\iota}_{2}\right) I=0 .
$$

Again, the solution is finite at the points $\lambda_{i^{\prime}}=0, \lambda_{j^{\prime}} \neq 0, i^{\prime} \neq j^{\prime}$, and $\lambda_{1}=i_{2}=0$. Note that there are two different directions along which the front can be approached.

Similarly, for the $A_{4}$ singularity

$$
F=\xi^{5}+i_{1} \xi^{3}+i_{2} \xi^{2}+\lambda_{3} \xi
$$

and we obtain the equation

$$
\left(5 \frac{\hat{\partial}^{4}}{\hat{\partial} i_{3}^{4}}-3 i_{1} \frac{\hat{\partial}}{\hat{\partial} i_{2}}+2 i i_{2} \frac{\partial}{\hat{\partial} \hat{i}_{3}}+\hat{i}_{3}\right) I=0,
$$

with the additional relations

$$
\begin{gathered}
\left(\frac{\hat{o}^{3}}{\partial \hat{\jmath}_{3}^{3}}+i \frac{\partial}{\partial \hat{\lambda}_{1}}\right) I=0, \\
\left(\frac{\hat{o}^{2}}{\partial \hat{i}_{2} \partial \hat{\iota}_{3}}-i \frac{\partial}{\partial \hat{\iota}_{1}}\right) I=0 .
\end{gathered}
$$

Let us finally discuss the $D_{4}$ singularity with

$$
F=\xi_{2}^{2} \xi_{1}-\xi_{1}^{3}+i_{2} \xi_{2}+i_{1} \xi_{1}+\lambda_{3} \xi_{1}^{2} \text {. }
$$

As this singularity depends on two normal slowness coordinates $\xi_{1}$, $\xi_{2}$, we find a system of two equations, viz.

$$
\begin{aligned}
\left(2 \frac{\partial^{2}}{\partial \lambda_{1} \partial \lambda_{2}}+\lambda_{1}\right) I & =0, \\
\left(\left(1-\lambda_{3}\right) \frac{\partial^{2}}{\partial \lambda_{1}^{2}}-3 \frac{\partial^{2}}{\partial \lambda_{2}^{2}}+\lambda_{2}\right) I & =0 .
\end{aligned}
$$

(Here we made use already one of the "vanishing" relations.) Without solving the equations we will determine their asymptotic behavior for vanishing versal parameters, i.e., the asymptotic expansion near the front.

Asymptotic Behavior of the Integrals. The fact that Picard-Fuchs equations are ordinary (matrix) differential equations with regular-singular points allows us to 
apply classical techniques to find the asymptotic behavior of the integrals for $\lambda=0$. To find the exact behavior we will rewrite (5.10) as a first-order matrix equation as follows. Define the $s \times s$ matrix

$$
A(\lambda)=\left(\begin{array}{cccccc}
0 & 1 & 0 & \cdots & \cdots & 0 \\
0 & 0 & 1 & 0 & \cdots & 0 \\
\vdots & \vdots & \ddots & \ddots & \ddots & \vdots \\
\vdots & \vdots & & \ddots & \ddots & 0 \\
0 & 0 & \cdots & \cdots & 0 & 1 \\
-q_{0}(\lambda) & -q_{1}(\lambda) & \cdots & \cdots & \cdots & -q_{s-1}\left(\lambda_{0}\right)
\end{array}\right),
$$

then the following matrix equation is equivalent to $(5.10)$

$$
i \frac{d}{d i} \Omega\left(i_{i}\right)=A(i) \Omega\left(i_{i}\right)
$$

with

$$
\Omega(\hat{\imath})=\left(\begin{array}{c}
\frac{P d \xi}{\partial \xi_{n} H} \\
i \frac{d}{d \lambda} \frac{P d \xi}{\partial \xi_{n} H} \\
\vdots \\
\left(\lambda \frac{d}{d \lambda}\right)^{s-1} \frac{P d \xi}{\partial_{\xi_{n}} H}
\end{array}\right)
$$

Standard theory now implies that around $\lambda=0$ the solution can be written in terms of a matrix $\Xi$ whose columns provide a basis in the $s$-dimensional solution space

$$
\Xi(\hat{i})=B(\hat{i}) \hat{\iota}^{M},
$$

where $B(\hat{\lambda})$ is a $s \times s$-matrix regular at $\lambda=0$ and $M$ is a constant $s \times s$ matrix. The expression $\lambda^{M}$ is defined as (for small $\lambda$ )

$$
\hat{\imath}^{M}=\mathbf{I}+M(\log \hat{\imath})+\frac{(M \log \hat{i})^{2}}{2 !}+\cdots .
$$

The matrix $B$ has a regular power series in $\lambda$ with constant coefficient matrices, which is absolutely convergent near $i=0$.

The monodromy resulting from going around the critical point at $\lambda=0$ is with respect to the basis defined by $\Xi$ given by the matrix $\exp (2 \pi i M)$. (This is the operator $h_{*}=h_{1} \cdot h_{2}$ defined in the previous section.) It is easy to see that it is a unipotent operator, i.e., there is an index $m_{0}$ such that $(\exp (2 \pi i M)-\mathbf{I})^{m_{0}}=0$. For the $A_{k}$-type singularities this index is equal to $m_{0}=\mu$, the multiplicity of the singularity.

As a result it follows that (see [30] for a general proof) close to the point $\lambda=0$, i.e., close to the intersection point with the singular set $\Sigma, I(\lambda)$ has the following general asymptotic expansion:

$$
I_{i}(\hat{\imath})=\sum_{q, \ell} a_{q, \lambda^{i}} \lambda^{q}(\log \lambda)^{\prime} .
$$

This series is absolutely convergent if $|\hat{i}|$ is small. The coefficients $a_{q, f}$ of the series are vectors in $\mathbb{C}^{\mu}$. The numbers $q$ are non-negative rational numbers; they 
have the property that $\exp (2 \pi i q)$ is an eigenvalue of the monodromy operator $h_{*}$. This property shows explicitly the relation between the asymptotic expansion and the monodromy group of the singularity. All coefficients $a_{q,}$ vanish for $\ell>0$.

As an example, consider the $A_{2}$ singularity discussed earlier. The eigenvalues of $h_{*}$ are then $\exp ( \pm 2 \pi i / 6)$ and the above result implies that close to $\lambda_{*}=0$,

$$
I=\sum_{r=0}^{\infty} a_{r} \lambda^{1 / 6+r}+\sum_{r=0}^{\infty} b_{r} \lambda^{5 / 6+r}
$$

This result agrees with the well-known high-frequency expansion of the fundamental solution in terms of the Airy function.

The leading exponent in expansions like (5.42) is referred to as the singularity index. Its relation to the order of the monodromy group has been extensively discussed by Arnold [2], however, not from the point of view adopted in this paper. In fact, it is not hard to see that the general leading asymptotic formula for an isolated singularity defined by a quasi-homogeneous polynomial $F(\xi, \lambda)$ is given by

$$
I \sim i^{1 / 2-q^{\prime}}, \quad q^{\prime}=1 / 2-\sum d_{i} .
$$

For the $A_{k}$ singularities the formula for $q^{\prime}$ is given by $q^{\prime}=\frac{1}{2}-\frac{1}{k+1}$. Hence, $I \sim \lambda^{1 / 6}$ for $A_{2}, I \sim \lambda^{1 / 4}$ for $A_{3}$ and $I \sim \lambda^{3 / 10}$ for $A_{4}$. For the $D_{k}$ singularities it turns out that $q^{\prime}=\frac{k-2}{2 k-2}$, so $I \sim \lambda^{1 / 6}$ for $D_{4}$. Note that the energy of the wave gets localized in the singularities corresponding to the degenerate critical points in the phase function at higher frequencies. This phenomenon is at the origin of caustics. We have indicated this asymptotic behavior in Fig. 4.1 and Fig. 4.3.

Acknowledgement. We would like to thank J.J. Duistermaat and R. Burridge for their interest in this work and their many valuable comments. We also thank S.W. Guest for his assistance in preparing Figs. 3.1-3.3. This paper was published with the permission of Shell Research B.V. and Schlumberger Cambridge Research Limited.

\section{References}

1. Al'shits, V.I., Lothe, J.: Elastic waves in triclinic crystals. I. General theory and the degeneracy problem. Kristallografiya 24, 672-683 (1979); Al'shits, V.I., Lothe, J.: Elastic waves in triclinic crystals. II. Topology of polarization fields and some general theorems. Kristallografiya 24, 683-693 (1979)

2. Arnold, V.I.: Remarks on the stationary phase method and the Coxeter numbers. Russ. Math. Surv. 28, No. 5, 19-48 (1973)

3. Arnold, V.I.: Wave front evolution and equivariant Morse lemma. Comm. Pure and Appl. Math. 29, 557-582 (1976)

4. Arnold, V.I.: Surfaces defined by hyperbolic equations. Math. Zam. 44, 3-18 (1988)

5. Arnold, V.I.: On the interior scattering of waves defined by hyperbolic variational principles. J. Geom. Phys. 5, 305-315 (1988)

6. Arnold, V.I., Gusein-Zade, S.M., Varchenko, A.N.: Singularities of Differentiable Maps. Vol. I and II, Boston: Birkhäuser, 1988

7. Arnold, V.I.: Singularities of Caustics and Wave Fronts. Dordrecht: Kluwer Academic Publ., 1990

8. Atiyah, M.F., Bott, R., Gårding, L.: Lacunae for hyperbolic differential operators with constant coefficients I and II. Acta. Math. 124, 109-189 (1970), and Acta Math. 131, 145-206 (1973); Gårding, L.: Sharp fronts of paired oscillatory integrals. Publ. Res. Int. Math. Sci. 12 (1976) 
9. Auld, B.A.: Acoustic fields and waves in solids. New York: John Wiley, 1973

10. Braam, P.J., Duistermaat, J.J.: Normal forms of real symmetric systems with multiplicity. Indag. Mat. 4, 407-421 (1993)

11. Burridge, R.: Lacunas in two dimensional wave propagation. Proc. Camb. Phil. Soc. 63, 819825 (1967); Burridge, R.: The singularity on the plane lids of the wave surface of elastic media with cubic symmetry. Quart. J. Mech. Appl. Math. XX, 41-56 (1967); Burridge, R.: Lamb's problem for an anisotropic half-space. Quart. J. Mech. Appl. Math. XXIV, 81-98 (1971)

12. Burridge, R., Chadwick, P., Norris, A.N.: Fundamental elastodynamic solutions for anisotropic media with ellipsoidal slowness surfaces. Submitted to the Proceedings of the Royal Society (1992)

13. Cagniard, L.: Réflexion et réfraction des ondes séismiques progressives. Paris: Gauthier-Villars, 1939

14. Crampin, S., Yedlin, M.: Shear wave singularities of wave propagation in anisotropic media. J. Geophys. 49, 43-46 (1981)

15. Duff, G.F.D.: The Cauchy problem for elastic waves in an anisotropic medium. Phil. Trans. R. Soc. A. 252, 249-273 (1960)

16. Duistermaat, J.J.: Oscillatory integrals, Lagrange Immersions and Unfolding of singularities. Commun. Pure Appl. Math. 27, No. 2, 207-281 (1974); Fedoryuk, M.V.: The stationary phase method and pseudodifferential operators. Russ. Math. Surv. 26, No. 1, 65-115 (1972); Mishchenko, A.S., Shatalov, V.E., Sternin, B.Yu.: Lagrangian Manifolds and the Maslov Operator. Berlin, Heidelberg, New York: Springer, 1990; Kendall, J.-M., Thomson, C.J.: Maslov ray summation, pseudo-caustics, Lagrangian equivalence and transient seismic wave forms. Submitted to Geophys. J. Int. (1992)

17. Every, A.G.: General closed-form expressions for acoustic waves in elastically anisotropic solids. Phys. Rev B 22, 1746-1760 (1980)

18. Gel'fand, I.M., Shilov, G.E.: Generalized functions. Vol. I, New York: Academic Press, 1964

19. Griffiths, P.A.: On the periods of certain rational integrals, I. Ann. Math. 90, 460-495 (1969)

20. de Hoop, A.T.: A modification of Cagniard's method for solving seismic pulse problems. Appl. Sci. Res. B8, 349-356 (1960)

21. Gorman, A.D., Wells, R., Fleming, G.N.: Wave propagation and Thom's theorem. J. Phys. A: Math. Gen. 14, 1519-1531 (1981)

22. Hubral, P., Tygel, M.: Transient response from a planar acoustic interface by a new pointsource decomposition into plane waves. Geoph. 50, 766-774 (1985)

23. Hudson, J.A.: Wavespeed and attenuation of elastic waves in material containing cracks. G.J.R.A.S. 64, 133-150 (1981)

24. van der Hijden, J.H.M.T.: Propagation of transient elastic waves in stratified anisotropic media. Amsterdam: North Holland, 1987

25. John, F.: Plane waves and spherical means applied to partial differential equations. New York: Interscience Publishers Inc., 1955 (Chapter II); John, F.: Partial differential equations. Berlin, Heidelberg, New York: Springer, 1982

26. Katz, N.: Differential equations for periods. Publ. Math. I.H.E.S. 35, 71 (1968)

27. Leray, J.: Hyperbolic differential equations. The Institute for Advanced Study, Princeton, N.J., 1952

28. Saito, K.: On the periods of primitive integrals. Harvard Lecture Notes, Cambridge (1980); Blok, B., Varchenko, A.: Topological conformal field theory and the flat coordinates. Int. J. Mod. Phys. A7, 1467 (1992); Lerche, W., Smit, D.-J., Warner, N.P.: Differential equations for periods and flat coordinates in two-dimensional topological matter theories. Nucl. Phys. B 372, 87 (1991); Morrison, D.R.: Picard-Fuchs equations and mirror maps for hypersurfaces. In: Essays on Mirror Manifolds. Yau, S-T. (ed) Hongkong: International Press, 1992

29. Ludwig, D.: Exact and asymptotic solutions of the Cauchy problem. Comm. Pure Appl. Math. 13, 473-508 (1960)

30. Malgrange, B.: Intégrale asymptotique et monodromie. Ann. Sci. École Normale Supp. 4, No. 7, 405-430 (1974)

31. Milnor, J.: Singular Points of Complex Hypersurfaces. Princeton, NJ: Princeton University Press, 1968

32. Musgrave, M.J.P.: On the propagation of elastic waves in aelotropic media I. Proc. R. Soc. Lond. A 226, 339 (1954); idem II, Proc. R. Soc. Lond. A 226, 356 (1954); Miller, G.F., Musgrave, M.J.P.: On the propagation of elastic waves in aelotropic media III. Proc. R. Soc. 
Lond. A 236, 352 (1956); Musgrave, M.J.P.: Crystal acoustics. San Francisco: Holden Day, 1970

33. Musgrave, M.J.P.: On an elastodynamic classification of orthorhombic media. Proc. R. Soc. Lond. A 374, 401-429 (1981); Musgrave, M.J.P.: Acoustic axes in orthorhombic media. Proc. R. Soc. Lond. A 401, 131-143 (1985)

34. Nichols, D., Muir, F., Schoenberg, M.: Expanded Abstracts 59th Ann. Mtg. SEG., 471 (1989)

35. Norris, A.N.: A theory of pulse propagation in anisotropic elastic solids. Wave Motion 9, 509-532 (1987)

36. Payton, R.G.: Elastic wave propagation in transversely isotropic media. The Hague: Martinus Nijhoff Publishers, 1983

37. Payton, R.G.: Int. J. Engng. Sci. 13, 183 (1975); Payton, R.G.: Instituto Lombardo (Rend. Sci) A 108, 684 (1974)

38. Petrovsky, 1.: On the diffusion of waves and the lacunas for hyperbolic equations. Math. Sbo. 17 (59), 289-370 (1945)

39. Riesz, M.: L-intégrale de Riemann-Liouville et le problem de Cauchy. Acta Math. 81, 1-223 (1949)

40. Salmon, G.: Geometry of three dimensions. Dublin: Hodges, Foster and Figgis, 1882

41. Schoenberg, M., Muir, F.: A calculus for finely layered anisotropic media. Geoph. 54, 581-589 (1989); Hood, J., Schoenberg, M.: NDE of fracture-induced anisotropy. In: Review of Progress in Quantitative Nondestructive Evaluation, 2101-2108, New York: Plenum Press, 1992

42. Sommerfeld, A.: Über die Ausbreitung der Wellen in der drahtlosen Telegraphie. Ann. Physik 28, 665-737 (1909); Weyl, H.: Ausbreitung elektromagnetischer Wellen über einem ebenen Leiter. Ann. Physik 60, 481-500 (1919)

43. Vasil'ev, V.A.: Sharpness and the local Petrovskii condition for strictly hyperbolic operators with constant coefficients. Math. USSR Izv. 28, 233-273 (1987)

Communicated by A. Jaffe 